Pacific Journal of Mathematics

PLANE ELLIPTIC GEOMETRY OVER RINGS

Vol. 123, No. 2

April 1986 


\title{
PLANE ELLIPTIC GEOMETRY OVER RINGS
}

\author{
Frieder KNUUPPEl AND EdZARD SALOW
}

\begin{abstract}
The classical model of plane elliptic geometry is a sphere of the real affine space. The points of this model are the pairs of antipodal points of the sphere, and the lines are the great circles of the sphere. Right angles retain their ordinary meaning. This model is isomorphic to the real projective plane, where orthogonality on the set of lines is given by a symmetric bilinear form such that no line is orthogonal to itself.

In the present paper we attempt a foundation and a study of plane elliptic geometry over commutative rings.
\end{abstract}

\section{CONTENTS}

Introduction

Part I. Metric Planes over Rings . . . . . . . . . . . . . . . . . . . . . 339

Part II. Plane Elliptic Geometry in Terms of Reflections . . . . . . . . . . . . . . . . 347

$\$ 1$ The System of Axioms. The Main Theorem . . . . . . . . . . . . . . 347

$\$ 2$ Basic Conclusions . . . . . . . . . . . . . . . . . . . . . . 348

$\S 3$ The Elliptic Plane and the Pseudo-Plane . . . . . . . . . . . . . 353

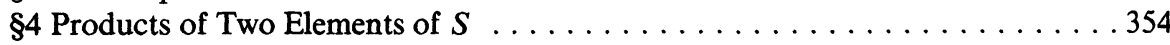

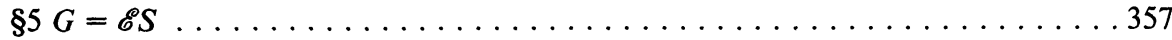

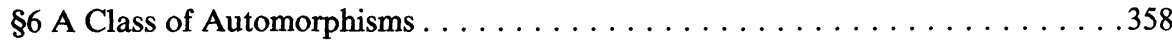

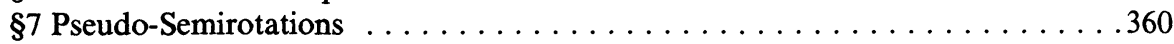

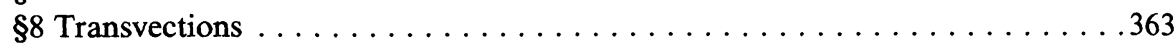

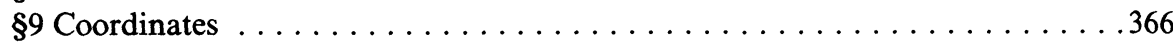

$\$ 10$ The Bilinear Form . . . . . . . . . . . . . . . . . . . . . 374

$\$ 11$ The Group of Motions . . . . . . . . . . . . . . . . . . . . . 379

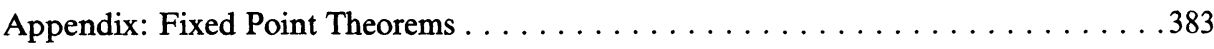

References . . . . . . . . . . . . . . . . . . . 384

Introduction. Let $R$ be a commutative ring with 1 . The points of the projective plane $\Pi(R)$ are the sets $R a$, where $a$ is a vector of the free $R$-module $R^{3}$ such that $a, b, c$ is a basis for some $b, c$. Replacing $R^{3}$ by the dual module $R^{3 *}$ one obtains the definition of a line $R g$ of $\Pi(R) . R a$ is incident with $R g$ if $a g=0$. Two different points need not have a common line, and they can have more than one common line.

Let $f: R^{3} \times R^{3} \rightarrow R$ be a symmetric bilinear form such that $(a, a) f$ is a unit for every point $R a$ of $\Pi(R)$. For every $a \in R^{3}$ let $a^{*}$ denote the linear function $R^{3} \rightarrow R, x \mapsto(a, x) f$. Then the homomorphism $R^{3} \rightarrow$ $R^{3 *}, a \mapsto a^{*}$ is a bijection; i.e. $f$ is an inner product in the sense of [6]. If 
$R a$ is a point then $R a^{*}$ is called the polar line of $R a$. A line is called orthogonal to $R a^{*}$ if and only if it passes through $R a$. The projective plane together with this polarity is called the elliptic plane $\Pi(R, f)$. The main purpose of Part II of our article is a synthetic approach to substructures of elliptic planes over rings.

Let $R a^{*}$ be a fixed line of $\Pi(R, f)$. Taking all points $R b$ such that $(a, b) f$ is a unit we obtain the point set of an affine plane $\Pi^{\prime}(R)$. If $R$ is a field then $\Pi^{\prime}(R)$ contains all points except the points of the line $R a^{*}$. But in general the affine plane will constitute a rather small part of the projective plane. This is one reason why the coordinatization of a (synthetically defined) elliptic plane involves difficulties.

In order to characterize pairs of points and lines being uniquely joined to each other we introduce a relation $\curvearrowleft R a \curvearrowleft R b$ means that $R b$ is an affine point when $R a^{*}$ is the line at infinity. In our system of axioms we use only one basic relation |, standing for incidence. The relation $\multimap$ is derived from | (compare [10], where $\longmapsto$ is a basic term). Therefore, however, we must restrict our study to commutative rings where every non-unit is a zero-divisor. Then two points $A, B$ satisfy $A \longmapsto B$ if and only if there is a pair of orthogonal lines $g, h$ such that $g$ is the unique perpendicular of $h$ through $A$, and $h$ is the unique perpendicular of $g$ through $B$. This property will supply the definition of $\multimap$ in our axiomatic approach. If $A \longmapsto B$ and $h$ is an arbitrary line through $B$ then there is exactly one orthogonal of $h$ through $A$. This property will be our first axiom. The second one is a richness condition, and the third one uses a three-reflection-theorem. A geometric property called (M) will not be used until we prove that 2 is a unit of the coordinate ring in the last section.

We need a weakened version of the property denoted by $\longmapsto$ which holds for arbitrary distinct points. Being unable to offer a suitable axiom that applies to any elliptic plane over a commutative ring, we use

(U) Given points $A, B$. Then there exists a line $h$ through $B$ such that exactly one line is incident with $A$ and orthogonal to $h$.

In an elliptic plane over a commutative ring of stable rank $\leq 3$ property (U) is valid. In particular (U) holds if any two points are incident with at least one common line. This plain geometric property would considerably facilitate our efforts. However we feel that this restriction is not adequate since it excludes too many rings.

In an elliptic plane each point and its polar line define a reflection. The basic concept of our system of axioms is a group $G$ together with a 
subset $S$ of involutions such that $S$ generates $G$. The elements of $S$ represent reflections, hence simultaneously also points and lines. Within this framework we formulate the axioms mentioned above.

The construction of a coordinate ring requires the existence of enough automorphisms of the geometric structure. Within our axiomatic approach the existence of sufficiently many suitable automorphisms need not be explicitly presupposed but can be proved.

Reflections of an elliptic plane are elements of its orthogonal group. Hence the study of elliptic geometry from our point of view is also a study of orthogonal groups over rings.

\section{PART I. Metric Planes over Rings}

Let $R$ be a commutative ring with 1 . Then the rank of a free $R$-module is unique. Let $R^{3}$ denote the free 3-dimensional $R$-module $R \times R \times R$. We write $R^{3 *}:=\operatorname{Hom}\left(R^{3}, R\right) . R x$ is called a point (a line) if $x, y, z$ is a basis of $R^{3}$ (of $R^{3 *}$ ) for some $y, z$. The point $R x$ is incident with the line $R y$ if $x y=0$; notation: $R x \mathrm{I} R y$ or $R y \mathrm{I} R x$. Let $\mathscr{P}(R)$ denote the set of points and $\mathscr{L}(R)$ the set of lines. $\Pi(R):=(\mathscr{P}(R), \mathscr{L}(R), \mathrm{I})$ is called the projective plane over $R$. We shall write $\mathscr{P}=\mathscr{P}(R)$ and $\mathscr{L}=$ $\mathscr{L}(R)$.

Every linear bijection $R^{3} \rightarrow R^{3}$ induces a contragredient mapping $R^{3 *} \rightarrow R^{3 *}$. This pair of mappings induces an automorphism (collineation) of $\Pi(R)$.

Let $R x, R y \in \mathscr{P}$. We write $R x$ distant $R y$ if $x, y, z$ is a basis of $R^{3}$ for some $z$. The analogous definition applies to a pair of lines. Take $R x \in \mathscr{P}$ and $R y \in \mathscr{L}$. We say $R x$ distant $R y$ or $R y$ distant $R x$ if $x y \in R *$ (group of units).

Next we collect some elementary lemmas. From each of them a dual counterpart can be obtained by interchanging the words "point" and "line". I.1 and I.3 can be found in [7] and in [10]. Proofs of I.2 and I.5 are given in [10]. I.4 is due to [2].

I.1. Let $A, B \in \mathscr{P}$. If $A$ distant $B$ then $A, B$ lie on exactly one line $g$. We write $g=(A, B)$.

1.2. The following statements are equivalent.

(i) $A$ distant $B$ if and only if $A, B$ have a unique common line.

(ii) Every non-unit of $R$ is a zero-divisor. 
I.3. Given $A \in \mathscr{P}$ and $g \in \mathscr{L}$. The following statements are equivalent.

(i) $A$ distant $g$.

(ii) $A$ distant $B$ and $(A, B)$ distant $g$ for any point $B \mathrm{I} g$.

(iii) $A$ distant $B$ and $(A, B)$ distant $g$ for some point $B \mathrm{I} g$.

An $n$-tuple $\left(\alpha_{1}, \ldots, \alpha_{n}\right) \in R^{n}$ is called unimodular if $R \alpha_{1}$ $+\cdots+R \alpha_{n}=R$. In other words, $\beta_{1} \alpha_{1}+\cdots+\beta_{n} \alpha_{n}=1$ for some $\beta_{i} \in$ $R$. Another equivalent statement is that $a b=1$ for some $b \in R^{n *}$, where $a=\left(\alpha_{1}, \ldots, \alpha_{n}\right)$.

I.4. Any two points have at least one common line if and only if the following condition holds. Let $(\alpha, \beta) \in R \times R$. Then $(\alpha, \beta)=(\lambda \gamma, \lambda \delta)$ for some $\lambda \in R$ and some unimodular $(\gamma, \delta) \in R \times R$.

\section{I.5. The following statements are equivalent}

(i) Let $A \in \mathscr{P}$ and $g, h \in \mathscr{L}$ with $A \mathrm{I} g, h$. Then $j$ distant $g, h$ for some $j \in \mathscr{L}$ with $j \mathrm{I} A$.

(ii) $R$ has stable rank 2 ; i.e. if $(\alpha, \beta) \in R \times R$ is unimodular then $\alpha+\beta \gamma$ is unimodular for some $\gamma \in R$ (i.e. $\alpha+\beta \gamma \in R^{*}$ ).

I.6. Suppose that $R$ has stable rank $\leq 3$; i.e. if $(\alpha, \beta, \gamma) \in R \times R \times R$ is unimodular then $(\alpha+\mu \gamma, \beta+\nu \gamma)$ is unimodular for some $\mu, \nu \in R$. Then for every $A \in \mathscr{P}$ and $g \in \mathscr{L}$ there is a line $h$ such that $h \mathrm{I} A$ and $h$ distant $g$.

Proof. Every bijective linear mapping of $R^{3}$ induces an automorphism of $\Pi(R)$. Thus we may assume $g=R[0,0,1], A=R(\alpha, \beta, \gamma)$. A set $h=R[\chi, \omega, \eta] \subseteq R^{3 *}$ is a line with the asserted properties if and only if $(+) \quad(\chi, \omega)$ is unimodular and $\alpha \chi+\beta \omega+\gamma \eta=0$.

The triple $(\alpha, \beta, \gamma)$ is unimodular since $A$ is a point. By our assumption, $(\alpha+\mu \gamma, \beta+\nu \gamma)$ is unimodular for some $\mu, \nu \in R$. Let $\chi:=-\beta-\nu \gamma$, $\omega:=\alpha+\mu \gamma, \eta:=\nu \alpha-\mu \beta$. Then $(+)$ holds.

A substructure $\Pi^{\prime}$ of an incidence structure $\Pi$ is called locally complete if each line of $\Pi$ which is incident with a point of $\Pi^{\prime}$ is a line of $\Pi^{\prime}$.

I.7. Let $u \in \mathscr{L}$. Define $\mathscr{P}^{\prime}:=\{A \in \mathscr{P}: A$ distant $u\}$ and $\mathscr{L}^{\prime}:=\{g$ $\in \mathscr{L}: g$ distant $u\}$. Then the substructure $\Pi^{\prime}:=\left(\mathscr{P}^{\prime}, \mathscr{L}^{\prime}, \mathrm{I}\right)$ of $\Pi=\Pi(R)$ is locally complete. For $g, h \in \mathscr{L}^{\prime}$ define $g \| h$ (parallel) if $(g, u)=(h, u)$. 
Then for every $A \in \mathscr{P}^{\prime}$ and $g \in \mathscr{L}^{\prime}$ there is a unique $h \in \mathscr{L}^{\prime}$ with $h \| g$ and $h \mathrm{I} A$.

The assertion follows immediately from I.1 and I.3. $\Pi^{\prime}$ is called the affine plane (related to the line $u$ ).

EXAmple. Let $u=R[0,0,1]$. Then $\mathscr{P}^{\prime}=\{R(\alpha, \beta, 1): \alpha, \beta \in R\}$ and $\mathscr{L}^{\prime}=\{R[\alpha, \beta, \gamma]: \alpha, \beta, \gamma \in R$ and $(\alpha, \beta)$ unimodular $\}$.

Let $f: R^{3} \times R^{3} \rightarrow R$ be a symmetric bilinear form. In the sequel we assume $2 \in R^{*}$. Call lines $R g, R h$ orthogonal if $(g, h) f=0$. For $A \subseteq R^{3}$ let $A^{\perp}:=\left\{x \in R^{3}:(a, x) f=0\right.$ for every $\left.a \in A\right\}$.

If $a \in R^{3}$ satisfies $(a, a) f \in R^{*}$ then $R^{3}=R a \oplus R a^{\perp}$, and

$$
\sigma_{R a}: R^{3} \rightarrow R^{3}, \quad x \mapsto-x+2 \frac{(x, a) f}{(a, a) f} a
$$

is the linear injective mapping with $a \mapsto a$ and $x \mapsto-x$ for $x \in R a^{\perp}$. $\sigma_{R a}$ is called the reflection in $R a . \sigma_{R a}$ is an involution and satisfies $\left(x \sigma_{R a}, y \sigma_{R a}\right) f=(x, y) f$ for $x, y \in R^{3}$, i.e. $\sigma_{R a}$ is orthogonal. $\sigma_{R a}=\sigma_{R b}$ implies $R a=R b$.

I.8. Given $a, b \in R^{3}$ with $(a, a) f,(b, b) f \in R^{*}$. The following statements are equivalent.

(i) $\sigma_{R a}$ and $\sigma_{R b}$ commute.

(ii) $a \sigma_{R b}=\lambda$ a for some $\lambda \in R$ with $\lambda^{2}=1$.

(iii) $\lambda_{1} a \in R b$ and $\lambda_{2} a \in R b^{\perp}$ for some $\lambda_{1}, \lambda_{2} \in R$ with $\lambda_{1}^{2}=\lambda_{1}$, $\lambda_{2}^{2}=\lambda_{2}, \lambda_{1}+\lambda_{2}=1$ and $\lambda_{1} \lambda_{2}=0$.

(iv) $a \sigma_{R b} \in R a$.

Proof. Let $c:=a \sigma_{R b}$. Then $(*) \sigma_{R b} \sigma_{R a} \sigma_{R b}=\sigma_{R c}$ (i) $\Rightarrow$ (ii). $\left(a \sigma_{R b}\right) \sigma_{R a}$ $=a \sigma_{R b}$ implies $a \sigma_{R b}=\lambda a$ for some $\lambda \in R$. Furthermore, $\lambda^{2}=1$ as $(a, a) f=\lambda^{2}(a, a) f$. (ii) $\Rightarrow$ (iii). We have $\lambda^{2}=1$ and $a \sigma_{R b}=\lambda a$. Let $\lambda_{1}:=\frac{1}{2}(1+\lambda)$ and $\lambda_{2}:=\frac{1}{2}(1-\lambda)$. Then $\lambda_{1} a \in R b$ and $\lambda_{2} a \in R b^{\perp}$. (iii) $\Rightarrow$ (iv). (iii) implies $a \sigma_{R b}=\left(\lambda_{1} a+\lambda_{2} a\right) \sigma_{R b}=\lambda_{1} a-\lambda_{2} a=$ $\left(\lambda_{1}-\lambda_{2}\right) a \in R a$, hence $R c=R a$ since $\sigma_{R b}$ is orthogonal. (iv) $\Rightarrow$ (i) follows immediately from $(*)$.

I.9. Let $(a, a) f,(b, b) f,(c, c) f \in R^{*}$. The identity $\sigma_{R a} \sigma_{R b}=\sigma_{R c}$ holds if and only if any two of the vectors $a, b, c$ are orthogonal. Then $a, b, c$ is $a$ basis of $R^{3}$. 
Proof. Let us assume that $a, b, c$ are pairwise orthogonal. Define $a^{*} \in R^{3 *}, x \mapsto(x, a) f$ for $x \in R^{3}$; likewise $b^{*}, c^{*}$. We have $a a^{*} \in R^{*}$ and $b a^{*}=0=c a^{*}$. Let $A$ denote the matrix whose rows are the coordinate vectors $a, b, c$, and let $B$ denote the matrix whose columns are the coordinate vectors of $a^{*}, b^{*}, c^{*}$ in the dual basis. Obviously,

$$
A B=\left(\begin{array}{ccc}
a a^{*} & & \\
& b b^{*} & \\
0 & & c c^{*}
\end{array}\right),
$$

hence $A$ is invertible. This means that $a, b, c$ is a basis of $R^{3}$. We have $a \sigma_{R a} \sigma_{R b}=-a=a \sigma_{R c}, b \sigma_{R a} \sigma_{R b}=b \sigma_{R b} \sigma_{R a}=b \sigma_{R a}=-b=b \sigma_{R c}$ (cf. I.8 (iii) $\Rightarrow$ (i)), $c \sigma_{R a} \sigma_{R b}=c=c \sigma_{R c}$. Therefore $\sigma_{R a} \sigma_{R b}=\sigma_{R c}$.

Conversely, assume $\sigma_{R a} \sigma_{R b}=\sigma_{R c}$. The reflections $\sigma_{R a}, \sigma_{R b}$ and also $\sigma_{R b}, \sigma_{R c}$ commute. From I.8(iii) we obtain idempotent elements $\lambda, \mu \in R$ such that $\lambda a \in R c^{\perp}$ and $(1-\lambda) a \in R c, \mu b \in R c^{\perp}$ and $(1-\mu) b \in R c$. Pick $x \in R c^{\perp}$. The assumption yields $-x \sigma_{R b}=x \sigma_{R a}$, hence

$$
x=\frac{(b, x) f}{(b, b) f} b+\frac{(a, x) f}{(a, a) f} a .
$$

Furthermore, $((1-\mu) b, x) f=0$, thus $(b, x) f=\mu(b, x) f$. Also $(a, x) f=$ $\lambda(a, x) f$. We conclude $x \in R \mu b+R \lambda a$. Thus we proved $R c^{\perp} \subseteq R \mu b+$ $R \lambda a$, hence $R^{3}=R c \oplus R c^{\perp} \subseteq R c+R \mu b+R \lambda a$. Therefore $c, \mu b, \lambda a$ is a basis of $R^{3}$. This implies $\mu, \lambda \in R^{*}$. Finally, since $\lambda$ and $\mu$ are idempotent, $\mu=1=\lambda$ and $a, b \in R c^{\perp}$.

I.10. Let $\sigma_{R a^{\prime}}=\sigma_{R a} \sigma_{R e}, \sigma_{R b^{\prime}}=\sigma_{R b} \sigma_{R e}, \sigma_{R c^{\prime}}=\sigma_{R c} \sigma_{R e}$. Then $\sigma_{R a} \sigma_{R b} \sigma_{R c}$ $=\sigma_{R d}$, where $d:=(b, c) f \cdot a-(a, c) f \cdot b+(a, b) f \cdot c . \sigma_{R d} \sigma_{R e}$ is a reflection. Furthermore, if $R a$ distant $R b$ then $R c$ distant $R d$.

Proof. Let $d^{\prime}:=\left(b^{\prime}, c^{\prime}\right) f \cdot a^{\prime}-\left(a^{\prime}, c^{\prime}\right) f \cdot b^{\prime}+\left(a^{\prime}, b^{\prime}\right) f \cdot c^{\prime}$. Let $s:=-(a, c) f \cdot b+(a, b) f \cdot c$. Then $s \in R a^{\perp} \cap R e^{\perp}$; cf. I.9. Since $a, a^{\prime}$, $e$ is an orthogonal basis (cf. I.9), $R a^{\perp} \cap R e^{\perp}=R a^{\prime}$. Also $s^{\prime} \in R a$, where $s^{\prime}$ is defined correspondingly to $s$. Thus $\left(s, s^{\prime}\right) f=0$. This implies

$$
(a, c) f \cdot\left(a^{\prime}, b^{\prime}\right) f \cdot\left(b, c^{\prime}\right) f+(a, b) f \cdot\left(a^{\prime}, c^{\prime}\right) f \cdot\left(c, b^{\prime}\right) f=0 .
$$

Two similar equations arise from cyclic permutations of $a, b, c$. These equations immediately imply $\left(d, d^{\prime}\right) f=0$. For $x_{1}:=a, x_{2}:=b$ and $x_{3}:=c$ Gram's determinant $G:=\operatorname{det}\left(\left(x_{i}, x_{k}\right) f\right)$ is zero, because $a, b, c \in$ $R e^{\perp}=R a+R a^{\prime}$. Hence $(d, d) f=(a, a) f \cdot(b, b) f \cdot(c, c) f-G \in R^{*}$. 
Also $\left(d^{\prime}, d^{\prime}\right) f \in R^{*}$. Consequently $e, d, d^{\prime}$ is an orthogonal basis; cf. I.9. We assert that $\sigma_{R d}$ and $\sigma_{R a} \sigma_{R b} \sigma_{R c}$ coincide on $\left\{e, d, d^{\prime}\right\}$. Both of the mappings fix $R e$. We have $d \sigma_{R a}=(b, c) f \cdot a+(a, c) f \cdot b-(a, b) f \cdot c$ since $-(a, c) f \cdot b+(a, b) f \cdot c \in R a^{\perp}$. Two similar arguments yield $d \sigma_{R a} \sigma_{R b} \sigma_{R c}=d=d \sigma_{R d}$. Similarly

$$
d^{\prime} \sigma_{R a} \sigma_{R b} \sigma_{R c}=-\left(b^{\prime}, c^{\prime}\right) f \cdot a^{\prime}+\left(a^{\prime}, c^{\prime}\right) f \cdot b^{\prime}-\left(a^{\prime}, b^{\prime}\right) f \cdot c^{\prime}
$$

since

$$
-\left(a^{\prime}, c^{\prime}\right) f \cdot b^{\prime}+\left(a^{\prime}, b^{\prime}\right) f \cdot c^{\prime} \in R e^{\perp} \cap R a^{\prime \perp}=R a .
$$

Finally we obtain $d^{\prime} \sigma_{R a} \sigma_{R b} \sigma_{R c}=-d^{\prime}=d^{\prime} \sigma_{R d}$.

Now let us assume in addition $R a$ distant $R b$. I.9 yields $e^{\perp}=R a+$ $R b$, and $a, b, e$ is a basis of $R^{3}$. Hence $c=\lambda a+\mu b$ for some $\lambda, \mu \in R$. The coordinates of the vectors $c, d, e$ in the basis $a, b, e$ are

$$
\begin{aligned}
& \lambda, \mu, 0 \\
& (b, c) f+\lambda(a, b) f,-(a, c) f+\mu(a, b) f, 0 \\
& 0,0,1,
\end{aligned}
$$

respectively. The matrix $M$ consisting of these three rows satisfies

$$
\operatorname{det} M=-\lambda(a, c) f-\mu(b, c) f=-(c, c) f \in R^{*} .
$$

Therefore, $c, d, e$ is a basis. In particular, $R c$ distant $R d$.

I.11. Definitions. For any $a \in R^{3}$ let $a^{*} \in R^{3 *}$ denote the mapping $x \mapsto(a, x) f$. We define a sub-structure $\Pi(R, f)=(\mathscr{P}(R, f), \mathscr{L}(R, f)$, I) of $\Pi(R)$ :

$$
\begin{aligned}
R a \in \mathscr{P}(R, f) \Leftrightarrow & a, b, c \text { is a regular orthogonal } \\
& \text { basis of } R^{3} \text { for some } b, c .
\end{aligned}
$$

Regular means that $(a, a) f,(b, b) f,(c, c) f \in R^{*}$.

$$
R g \in \mathscr{L}(R, f) \Leftrightarrow g=a^{*} \text { for some } R a \in \mathscr{P}(R, f) .
$$

Let $R a \in \mathscr{P}(R, f)$. The line $R a^{*}$ is called the polar of $R a$, and $R a$ is called the pole of the line $R a^{*}$. The pair of mappings: $R a \mapsto R a^{*}$ for $R a \in \mathscr{P}(R, f)$ and $R a^{*} \mapsto R a$ for $R a^{*} \in \mathscr{L}(R, f)$ is a polarity of $\Pi(R, f)$. A pair of points $R a, R b \in \mathscr{P}(R, f)$ (a pair of lines $R a^{*}, R b^{*} \in$ $\mathscr{L}(R, f))$ is called orthogonal if $R a \mathrm{I} R b^{*}$.

1.12. Let $R a \in \mathscr{P}(R, f)$ and $R b^{*} \in \mathscr{L}(R, f)$. Then $R a \mathrm{I} R b^{*}$ if and only if $a, b, c$ is a regular orthogonal basis of $R^{3}$ for some $c$. 
Proof. If $a, b, c$ is an orthogonal basis then $(a, b) f=0$, hence $R a \mathrm{I} R b^{*}$. Conversely let us assume $R a \mathrm{I} R b^{*}$, i.e. $(a, b) f=0 . R a$ distant $R a^{*}$ (since $\left.a a^{*}=(a, a) f \in R^{*}\right)$ and $R a \mathrm{I} R b^{*}$ imply $R b^{*}$ distant $R a^{*}$; cf. I.3(i) $\Rightarrow$ (ii). Hence $R c \mathrm{I} R b^{*}, R a^{*}$ for some $R c \in \mathscr{P}(R)$; cf. I.1. Any two of the vectors $a, b, c$ are orthogonal. From $R a$ distant $R a^{*}$ and $R b$, $R c \mathrm{I} R a^{*}$ and $R b$ distant $R c$ follows that $a, b, c$ is a basis of $R^{3}$.

I.13. Let $\mathscr{S}(R, f):=\left\{\sigma_{R a}: R a \in \mathscr{P}(R, f)\right\}$. For $R a, R b \in \mathscr{P}(R, f)$ the following statements are equivalent.

(i) $\sigma_{R a} \sigma_{R b} \in \mathscr{S}(R, f)$.

(ii) $R a \mathrm{I} R b^{*}$.

(iii) $R a^{*} \perp R b^{*}$.

(iv) $R a \perp R b$.

(v) $(a, b) f=0$.

(vi) $a, b, c$ is a regular orthogonal basis of $R^{3}$ for some $c$. This is obvious from I.9 and I.12.

\section{I.14. $\Pi(R, f)$ is called an elliptic plane if $\Pi(R, f)=\Pi(R)$.}

REMARK. $\Pi(R, f)$ is an elliptic plane if and only if any homomorphism of $R$ onto a field $\bar{R}$ induces a homomorphism of $\Pi(R, f)$ such that the image $\Pi(\bar{R}, \bar{f})$ is an elliptic plane in the usual sense.

LEMMA. $\Pi(R, f)=\Pi(R)$ if and only if $(a, a) f \in R^{*}$ for every point Ra of $\Pi(R)$.

Proof. Let $(a, a) f \in R^{*}$ for every basis $a, b, c$ of $R^{3}$. Given $R a \in$ $\mathscr{P}(R)$. The usual vector space method can be applied in order to construct an orthogonal basis $a, b, c$. Hence $R a \in \mathscr{P}(R, f)$. The mapping $R a \mapsto$ $R a^{*}$ is a bijection of $\mathscr{P}(R)$ into $\mathscr{L}(R)$ since a regular orthogonal basis of $R^{3}$ exists. Therefore $\mathscr{L}(R)=\mathscr{L}(R, f)$.

I.15. Let $R a, R b \in \mathscr{P}(R, f)$. We write $\sigma_{R a} \mid \sigma_{R b}$ if $\sigma_{R a} \sigma_{R b} \in \mathscr{S}(R, f)$; cf. I.13. If $\sigma_{R a}, \sigma_{R b} \mid \sigma_{R c}$ for a unique $\sigma_{R c} \in \mathscr{S}(R, f)$ then we write $\sigma_{R a} \mathbf{u} \sigma_{R b}$. The abbreviation $\sigma_{R a}-\sigma_{R b}$ is to denote that $\sigma_{R a}\left|\sigma_{R c} ; \sigma_{R c}\right| \sigma_{R d} ; \sigma_{R d} \mid \sigma_{R b}$ and $\sigma_{R a} \mathbf{u} \sigma_{R d}$ and $\sigma_{R c} \mathbf{u} \sigma_{R b}$ for some $\sigma_{R c}, \sigma_{R d} \in \mathscr{S}(R, f)$.

Proposition. Suppose that every non-unit of $R$ is a zero-divisor and $(a, a) f \in R^{*}$ for every $R a \in \mathscr{P}(R)$ (hence $\Pi(R, f)=\Pi(R)$; cf. I.14).

(i) $R a \mathrm{I} R b^{*} \Leftrightarrow \sigma_{R a} \mid \sigma_{R b} \Leftrightarrow R a \perp R b \Leftrightarrow R a^{*} \perp R b^{*} \Leftrightarrow f(a, b)=0$. 
(ii) $R a$ distant $R b \Leftrightarrow$ there is a unique line of $\Pi(R)=\Pi(R, f)$ joining $R a$ to $R b \Leftrightarrow R a^{*}$ and $R b^{*}$ intersect in just one point $\Leftrightarrow \sigma_{R a} \mathbf{u} \sigma_{R b}$.

(iii) $R a$ distant $R b^{*} \Leftrightarrow R a^{*}$ distant $R b \Leftrightarrow f(a, b) \in R^{*} \Leftrightarrow \sigma_{R a} \sqcap \sigma_{R b}$.

Proof. (i) and (ii) follow immediately from I.2 and I.13. The equivalence of the first three statements of (iii) is obvious from the definitions. The last one is a translation of I.3 (iii).

I.16. Suppose that every non-unit of $R$ is a zero-divisor, and that $(a, a) f \in R^{*}$ for every point $R a \in \mathscr{P}(R)$. Let $\mathscr{G}:=\mathscr{G}(R, f)$ denote the group generated by $\mathscr{S}:=\mathscr{S}(R, f)$. We denote the elements of $\mathscr{S}$ by lowercase letters and use the notations introduced in I.15: $a \mid b$ means $a b \in \mathscr{S}$, and $a \mathbf{u} b$ indicates that $c \mid a, b$ for just one $c . a \longmapsto b$ is to denote that $a|c ; c| d ; d \mid b$ and $a \mathbf{u} d$ and $c \mathbf{u} b$ for some $c, d$.

$\mathscr{S}$ is invariant under inner automorphisms of $\mathscr{G}$, and $(\mathscr{G}, \mathscr{S})$ satisfies the following properties.

(E1) If $a \rightsquigarrow b$ and $b \mid c$ then $a \mathbf{u} c$.

(E2) If $a \mid b$ then $c \mid a$ and $c \mathbf{u} b$ and $c \rightsquigarrow b$ for some $c$.

(E3) $a, b, c \mid e$ implies $a b c \in \mathscr{S}$.

(M) If $a \longmapsto b$ then $a b$ is not an involution.

Proof. (E1) follows from I.15(ii) and I.3(i) $\Rightarrow$ (ii). (E2). Let $\sigma_{R a} \mid \sigma_{R b}$, i.e. $\sigma_{R a} \sigma_{R b}=\sigma_{R d}$ for some $R d \in \mathscr{P}(R)$. Then $a, b, d$ is a regular orthogonal basis of $R^{3}$; cf. I.9. Thus, $a, b, c:=b+d$ is a basis of $R^{3}$. In particular, $R c \in \mathscr{P}(R)$ and $R c$ distant $R b$. Hence $\sigma_{R c} \mathbf{u} \sigma_{R b}$; cf. I.15(ii). $(b, c) f=(b, b) f \in R^{*}$ implies $\sigma_{R c} \multimap \sigma_{R b}$; cf. I.15(iii). (E3) follows from I.15(i) and I.10. (M). Let $\sigma_{R a} \multimap \sigma_{R b}$, hence $(a, b) f \in R^{*}$; cf. I.15(iii). Suppose that $\sigma_{R a}$ and $\sigma_{R b}$ commute. From I.8(iv) we have $a \sigma_{R b} \in R a$. Now the formula for $\sigma_{R b}$ immediately shows $R a=R b$, hence $\sigma_{R a}=\sigma_{R b}$.

REMARK. If $R$ has stable rank 3 (cf. I.6) then $(G, S)$ satisfies

(U) Let $a, b \in \mathscr{S}$. Then $c \mid a$ and $c \mathbf{u} b$ for some $c \in \mathscr{S}$.

$(\mathscr{G}, \mathscr{S})$ is called the group of motions of the elliptic plane.

Let $\mathscr{F}(R, f):=\left\{\alpha \in \operatorname{Aut}\left(R^{3}, f\right): \operatorname{det} \alpha=1\right.$ and $\left.\operatorname{det}(1+\alpha) \in R^{*}\right\}$. The following result is a "representation theorem" for $\mathscr{F}(R, f)$.

I.17. Let $(a, a) f \in R^{*}$ for every point $R a \in \mathscr{P}(R)$. Let $R p \in \mathscr{P}(R, f)$.

(a) Let $\alpha \in \mathscr{F}(R, f)$ and $R g^{*} \in \mathscr{L}(R, f)$ with $R g^{*} I R p$. Then $\alpha=$ $\sigma_{R q} \sigma_{R g} \sigma_{R h}$ for some $R q \in \mathscr{P}(R, f)$ and $R h^{*} \in \mathscr{L}(R, f)$, where $R h^{*} \mathrm{I} R p$ and $R g^{*}$ distant $R h^{*}$ and $R p$ distant $R q^{*}$. 
(b) Given $R q \in \mathscr{P}(R, f)$ and $R g^{*}, R h^{*} \in \mathscr{L}(R, f)$ such that $R g^{*}$, $R h^{*} \mathrm{I} R p$ and $R g^{*}$ distant $R h^{*}$ and $R p$ distant $R q^{*}$. Then $\alpha:=\sigma_{R q} \sigma_{R g} \sigma_{R h}$ $\in \mathscr{F}(R, f)$.

Proof of (a). Let $q:=p\left(\alpha^{-1}+1\right) . \operatorname{det}\left(\alpha^{-1}+1\right)=\operatorname{det} \alpha^{-1}$. $\operatorname{det}(1+\alpha) \in R^{*}$ implies $R q \in \mathscr{P}(R, f)$. Select $R j^{*} \in \mathscr{L}(R, f)$ with $R j^{*} \mathrm{I} R q$. Let $e=j(1+\alpha)$. Then $R e^{*} \in \mathscr{L}(R, f)$, and the equation

$$
0=(j, q) f=\left(j, p \alpha^{-1}\right) f+(j, p) f=(j(\alpha+1), p) f=(e, p) f
$$

implies $R e^{*} \mathrm{I} R p$.

$$
\begin{aligned}
R^{*} & \ni(q, q) f=\left(p \alpha^{-1}+p, p \alpha^{-1}+p\right) f \\
& =2\left(p, p\left(1+\alpha^{-1}\right)\right) f=2(p, q) f .
\end{aligned}
$$

Also, $R^{*} \ni(e, e) f=2(j, e) f$. Hence, $R p^{*}$ distant $R q$ and $R j$ distant $R e^{*}$. In particular, $R p^{*}$ distant $R j^{*}$, since $R j^{*} \mathrm{I} R q$. Thus, $R d^{*} \mathrm{I} R p, R j$ for a unique line $R d^{*} \in \mathscr{L}(R, f)$.

$$
\begin{aligned}
p \alpha^{-1} \sigma_{R q} & =\left(1 / 2 \cdot p\left(1+\alpha^{-1}\right)-1 / 2 \cdot p\left(1-\alpha^{-1}\right)\right) \sigma_{R q} \\
& =1 / 2 \cdot p\left(1+\alpha^{-1}\right)+1 / 2 \cdot p\left(1-\alpha^{-1}\right)=p,
\end{aligned}
$$

since $p\left(1+\alpha^{-1}\right)=q$ and $\left(p\left(1+\alpha^{-1}\right), p\left(1-\alpha^{-1}\right)\right) f=0$. Also $j \alpha \sigma_{R e}=$ $j$. Together with $p \sigma_{R e}=-p$ and $j \sigma_{R q}=-j$ we conclude $p \beta=-p$ and $j \beta=-j$, where $\beta=\sigma_{R q} \alpha \sigma_{R e}$. Therefore, $x \beta=-x$ for any $x \in R p+R j$ $=d^{\perp}$ (apply $R p$ distant $R j$ ). This yields $(R d) \beta=R d$, hence $d \beta=d$ as $\operatorname{det} \beta=1$. Thus we proved $\beta=\sigma_{R d}$, i.e. $\alpha=\sigma_{R q} \sigma_{R d} \sigma_{R e}$. From I.10 we obtain $\sigma_{R d} \sigma_{R e}=\sigma_{R g} \sigma_{R h}$, where $R h^{*}$ is a line with $R h^{*} I R p$ and $R g^{*}$ distant $R h^{*}$.

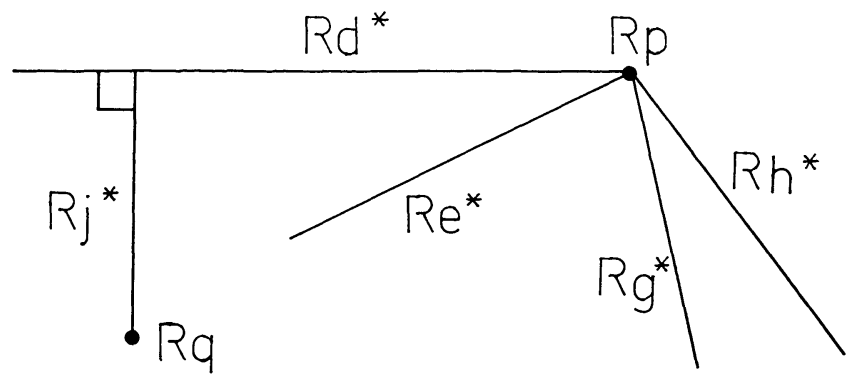

Proof of (b). det $\alpha=1$ as $\alpha$ is a product of reflections. We want to prove $\operatorname{det}(\alpha+1) \in R^{*}$. Select $q_{1}, q_{2}$ such that $q, q_{1}, q_{2}$ is an orthogonal basis. The assumption $R p$ distant $R q^{*}$ implies $R p$ distant $R q_{i}$. Let 
$R d_{i}^{*} \in \mathscr{L}(R, f)$ such that $R d_{i}^{*} I R p, R q_{i}$. Then $R d_{1}^{*}$ distant $R d_{2}^{*}$ (apply I.3, using $R p$ distant $R q^{*}$ and $R q_{1}$ distant $R q_{2}$ ). By I.10 there are lines

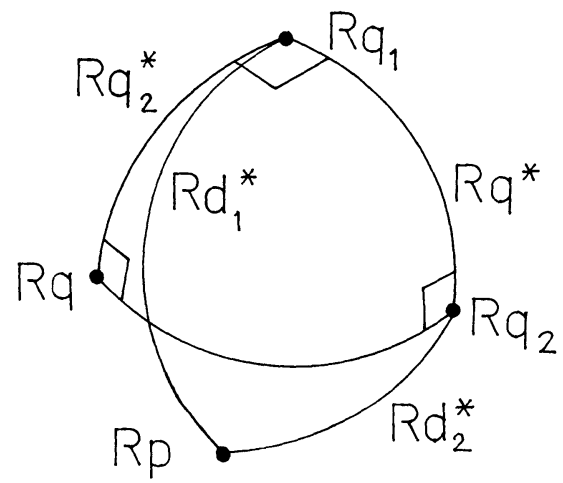

$R e_{i}^{*}$ through $R p$ such that $\sigma_{R g} \sigma_{R h}=\sigma_{R d_{i}} \sigma_{R e_{i}}$. The equation $\sigma_{R d_{1}} \sigma_{R d_{2}}=$ $\sigma_{R e_{1}} \sigma_{R e_{2}}$ and $R d_{1}$ distant $R d_{2}$ imply $R e_{1}$ distant $R e_{2}$; cf. I.10. Hence $R e_{1}+R e_{2}=p^{\perp}$. We have

$$
q_{i}(\alpha+1)=q_{i} \sigma_{R q} \sigma_{R g} \sigma_{R h}+q_{i}=-q_{i} \sigma_{R d_{i}} \sigma_{R e_{i}}+q_{i}=q_{i} \sigma_{R e_{i}}+q_{i}=\lambda_{i} e_{i},
$$

where $\lambda_{i}:=2\left(q_{i}, e_{i}\right) f\left(\left(e_{i}, e_{i}\right) f\right)^{-1} \in R^{*}$. Hence

$$
q^{\perp}(\alpha+1)=\left(R q_{1}+R q_{2}\right)(\alpha+1)=R e_{1}+R e_{2}=p^{\perp} \text {. }
$$

Finally,

$$
q(\alpha+1)=\left(q+q \sigma_{R p}\right) \sigma_{R g} \sigma_{R h}+\left(-q \sigma_{R p} \sigma_{R g} \sigma_{R h}+q\right)=\lambda p+w,
$$

where $w$ denotes the last bracket and $\lambda=2(q, p) f((p, p) f)^{-1} \in R^{*}$. From the reflection formula one infers that

$$
w \in 2 q-2 \frac{(q, p) f}{(p, p) f} p+R g+R h \subseteq p^{\perp} .
$$

We proved $R^{3}=R p+p^{\perp} \subseteq R^{3}(\alpha+1)$. Therefore $\operatorname{det}(\alpha+1) \in R^{*}$.

\section{PART II. Plane Elliptic Geometry in Terms of Reflections}

1. The system of axioms. The main theorem. Our system of axioms aims at elliptic planes over commutative rings. Under the assumptions of I.16 of Part I the group of motions of such an elliptic plane will satisfy our system of axioms, possibly apart from (U). If the ring has stable rank 3 then $(U)$ will be fulfilled, but we do not know a nice property of the ring which is equivalent to $(\mathrm{U})$. 
The basic assumption. The pair $(G, S)$ consists of a group $G=$ $\{\alpha, \beta, \ldots\}$ and $a$ set $S=\{a, b, \ldots\} \neq \varnothing$ of involutions such that $S$ is invariant under inner automorphisms of $G$ and $S$ generates $G$.

Notations. $a \mid b$ if $a b \in S$. Let $a \mathbf{u} b$ denote that there is a unique $c$ with $c \mid a, b$. Given $a, b$. If $a|c ; c| d ; d \mid b$ and $a \mathbf{u} d$ and $c \mathbf{u} b$ for some $c, d$ then we write $a \curvearrowleft b$. We say $c$ joins $a$ to $b$ if $c \mid a, b$. u, and $\mid$ are invariant under inner automorphisms.

\section{Axioms.}

(E1) If $a \rightsquigarrow b$ and $b \mid c$ then $a \mathbf{u} c$.

(E2) If $a \mid b$ then $c \mid a$ and $c \mathbf{u} b$ and $c \longmapsto b$ for some $c$.

(E3) $a, b, c \mid d$ implies $a b c \in S$

(M) If $a \longmapsto b$ then $a b$ is not an involution.

(U) Given $a, b$. Then $c \mid a$ and $c \mathbf{u} b$ for some $c$.

Our last axiom implies the following two statements.

$\left(\mathrm{U}^{\prime}\right)$ Let $a \in S$. Then $a \mid b$ for some $b$.

( $\left.\mathrm{U}^{\prime \prime}\right)$ Given $a, b$. Then $a|c ; c| d ; d \mid b$ for some $c, d$.

Axiom (M) will not be used until we study the group of motions in $\S 11$. Moreover, in $\S 2$ and $\S 3$ only (E1), (E2), (E3) and (U') will be used.

The main purpose of this article is the proof of the following theorem. Simultaneously, the proof is a study of the group of motions of an elliptic plane over a commutative ring.

TheOREM. Let $(G, S)$ satisfy the basic assumption and (E1), (E2), (E3), $(\mathrm{M})$ and $(\mathrm{U})$. Then there exist a commutative ring $R$, a bilinear form $f$ : $R^{3} \times R^{3} \rightarrow R$, and a mapping $\sigma$ with the following properties.

1. Every non-unit of $R$ is a zero-divisor, and 2 is a unit.

2. $\Pi(R, f)$ is an elliptic plane in the sense of Part $I$.

3. $\sigma$ is a monomorphism of the group $G$ into the group $\mathscr{G}$ such that $S \sigma \subseteq \mathscr{S}$, where $(\mathscr{G}, \mathscr{S})$ denotes the group of motions of $\Pi(R, f)$. Furthermore, $\mathscr{F}(R, f) \subseteq G \sigma$.

2. Basic concepts. In this section we assume that $(G, S)$ satisfies our basic assumption and (E1), (E2), (E3) and ( $\left.\mathrm{U}^{\prime}\right)$.

2.1. (a) $\mid, \longmapsto$ and $\mathbf{u}$ are symmetric relations on the set $S$. Furthermore, they are invariant under inner automorphisms of $G$.

(b) $a \mid b$ and $a, b \mid c$ implies $a b=c$.

(c) If $a \mid b$ then $a \mathbf{u} b$.

(d) $a \curvearrowleft a$ for every $a$. 
(e) $a \mid b$ and $a b c \in S$ implies $a b \mid c$.

(f) Let $d \longmapsto e$ and $a \mid d$ and $b \mid a, e$. The $a b \longmapsto d$,e.

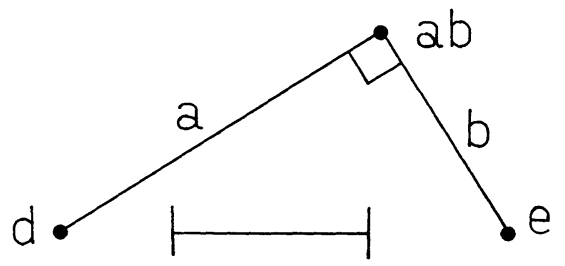

Proof. (a) and (e) are obvious. (b). Select $d$ such that $d \mid b, d \mathbf{u} a$ and $d \curvearrowleft a$; cf. (E2). (E1) implies $b \mathbf{u} a$. Thus, $a, b \mid c$ and $a, b \mid a b$ yields $c=a b$. (c) is a consequence of (b). (d). Select $b \in S$ such that $b \mid a$; cf. ( $\left.\mathrm{U}^{\prime}\right)$. Then $a|b ; b| a b ; a b \mid a$ and $a \mid a b$ and $b \mid a$. Thus, $a \curvearrowleft a$ by (c). (f). Our assumptions yield $d|a ; a| b ; b \mid a b$. (E1) implies $d \mathbf{u} b$. Finally, $a \mathbf{u} a b$ by (c). Hence we obtained $d \longmapsto a b$.

2.2. Let $a \mid b$ and $c \mid a b$. Then $a \mathbf{u} c$ if and only if $b \longmapsto c$. The figures indicate four possible interpretations of " $a \mid b$ and $c \mid a b$ ".
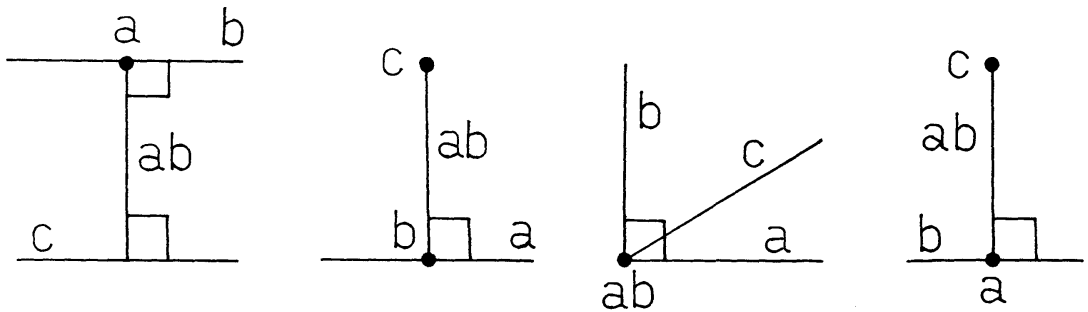

Proof. We have $b|a ; a| a b ; a b \mid c$ and $b \mathbf{u} a b$; cf. (c). Hence, $a \mathbf{u} c$ implies $b \longmapsto c$. Conversely, the assumption $b \longmapsto c$ together with $a \mid b$ yields $a \mathbf{u} c$; cf. (E1).

\subsection{Let $a, b, c \mid d$. Then $a b c \in S$ and $a b c \mid d$.}

Proof. $a b c \in S$ by (E3). Also $a, b, c d \in S$ and $a, b, c d \mid d$. Hence $a \cdot b \cdot c d \in S$ by (E3).

2.4. (i) Let $a, b \mid g$ and $a \mathbf{u} b$. Then $c \mid g$ and $c \mathbf{u} a, b$ for some $c$.

(ii) Let $a, b \mid g$ and $a \rightsquigarrow b$. Then $c \mid g$ and $c u a$ and $c-b$ for some $c$.

Proof. (i). Select $r, s$ with $r \mid a$ and $s \mid b$ and $r, s \mathbf{u} g$ and $r, s \curvearrowleft g$; cf. (E2). Then $r-b$ and $s \rightsquigarrow a$, hence $r u s$. Let $v \mid r, s$. We have $v \mathbf{u} a, b$ and 
conclude $v \longmapsto g$ and $v \mathbf{u} g$. Let $d|g, v . v| r ; r|a ; a| a g$ and $v \mathbf{u} a$ and $r \mathbf{u} a g$ (as $r \curvearrowleft g$ by 2.2) imply $v \curvearrowleft a g$. Hence $d \mathbf{u} a g$. Applying 2.2 twice we get $c:=d g \mathbf{u} a$ and, likewise, $c \mathbf{u} b$.

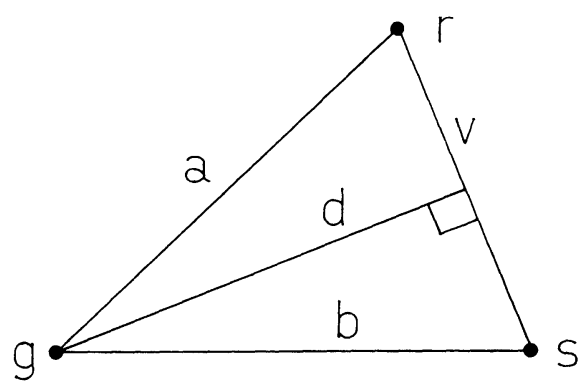

Proof of (ii). From 2.2 we know $a \mathbf{u} b g$. From (i) we obtain $c \mid g$ with $c \mathbf{u} a, b g$. Thus $c \mathbf{u} a$ and $c \longmapsto b$; cf. 2.2 .

2.5. If $a c=b d \in S$ and $a \mathbf{u} b$ then $c \mathbf{u} d$.

Proof. 2.2 yields $a \curvearrowleft b a c=d$, hence $c \mathbf{u} d$; cf. (E1).

2.6. Let $a, b \mid c$. If $d \mid a, b$ and $d \backsim c$ implies $d=c$ then $a \mathbf{u} b$.

Proof. We proceed in a number of steps.

(i) Let $a b=c d$ and $a, b, c, d \mid e$. Suppose that $g \mid c, d$ and $g \longmapsto e$ implies $g=e$. If $h \mid a, b$ and $h \longmapsto e$ then $h \mid e c$, ed.

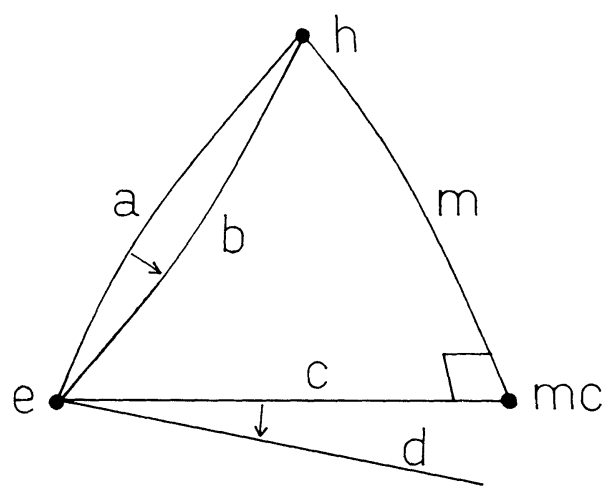

Proof of (i). (E1) implies $h \mathbf{u} c, d$. Let $m \mid c, h$. Since $m, a, b \mid h$, (E3) implies $m c d=m a b \in S$, i.e. $m c \mid c, d$. $h \longmapsto e$ yields $e \mathbf{u} m$; cf. (E1). There- 
fore, $e \mapsto m c$ by 2.2. Our assumptions imply $e=m c$, i.e. $e c=m \mid h$. Similarly ed $\mid h$.

(ii) Let $a b=c d$ and $a, b, c, d \mid e$. Suppose that $g \mid c, d$ and gセe implies $g=e$. Then $h \mid a, b$ and $h \longmapsto e$ implies $h=e$.

Proof. Let $h \mid a, b$ and $h \rightsquigarrow e$. Then $h \mid e c, e d$ by (i). Select $v$ such that $v \mid e$ and $v \mathbf{u} c$, ec; cf. 2.4 (i). (E3) implies $w:=v a b \in S$; furthermore, $w \mid e$ by 2.3 . We claim

$$
\text { If } f \mid v, w \text { and } f \curvearrowleft e \text { then } f=e \text {. }
$$

Namely, $f \mid v, w$ and $f \longmapsto e$ implies $f \mid e c, e d$ by (i) (take $v, w, f$ instead of $a$, $b, h)$. Hence $f, e \mid v, e c$, and the assumption vuec yields $f=e$. Thus, $(*)$ is true. Applying (i) once more, we obtain $h \mid e v, e w .2 .5$ implies evuec, since $e v \cdot v=e c \cdot c \in S$ and $v \mathbf{u} c$. Therefore, $h \mid e c, e v$ implies $h=e$.

Now we are ready to prove the proposition. Let $a, b \mid c$ and suppose that $a \mathbf{u} b$ is not true. Then $e \mid a, b$ for some $e \neq c$. Select $s$ with $s \mid e$ and $s \mathbf{u} a$ and $s \longmapsto a$; cf. (E2).

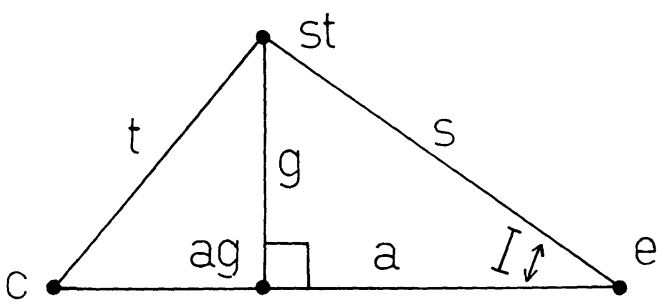

(E1) yields $c u s$. Let $t \mid c, s$. Then $p:=s a b \in S$ and $p \mid e$ by 2.3. The equation $t s p=t a b \in S$ shows $p \mid s t$, and $s \curvearrowleft a$ implies $s t u a$; cf. (E1). Let $g \mid s t, a$. Since $p, s, g \mid s t$, (E3) yields $r:=b a g=p s g \in S$, hence $b, r \mid a g$. Now $a \rightsquigarrow s$ implies $c u s$, hence $c \rightsquigarrow s t$ by 2.2. As $g \mid s t$, (E1) and 2.2 yield $c \mathbf{u} g$ and $c \rightsquigarrow a g$. Furthermore $a g \mid a, b$. The assumption in the proposition implies $a g=c$. In particular, $b, a, g \mid c$, hence $r \mid c$ by 2.3 . We have $a, b, g, r \mid c$ and $a b=g r$. Furthermore, $s t \mid g, r$ and $s t \mapsto c$ and $s t \neq c$ (the last statement is true, since $s t=c$ would imply $a, s \mid c, e$, contradicting $a \mathbf{u} s$ and $c \neq e$ ). We apply (ii) and obtain an element $d \neq c$ with $d \mid a, b$ and $d \mapsto c$. Thus we reach a contradiction.

Statement (ii) in the proof of 2.6 and the assertion of 2.6 yield 2.7. Let $a, b, c, d \mid e$ and $a b=c d$. If $a \mathbf{u} b$ then $c \mathbf{u} d$. 
2.8. Let $a \mid c, e$ and $b \mid d, e$. If $d \mathbf{u} e$ and $a \mathbf{u} b$ then $c \mathbf{u} d$.

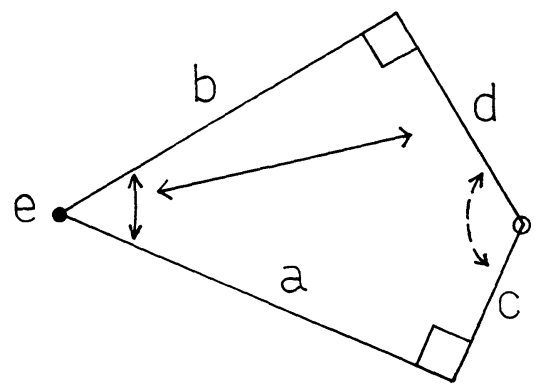

Proof. $a \mathbf{u} b$ and $e \mathbf{u} d$ mean $a \longmapsto d$ by definition. Hence $c \mathbf{u} d$.

2.9. Let $a, b \mid e$ and $c \mid a, f$ and $d \mid b, f$. If $e \backsim f$ and $a \mathbf{u} b$ then $c \mathbf{u} d$. This follows immediately from 2.8 .

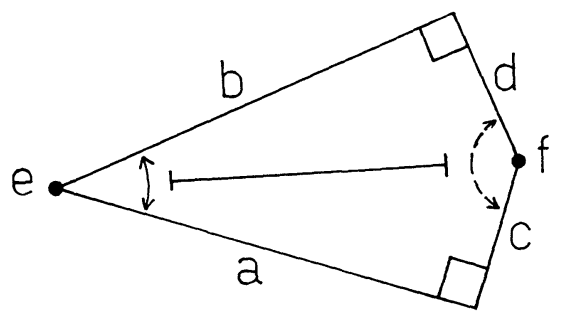

2.10. Let $e \mid a, b ; a b c \in S ; a \mathbf{u} b$ and $c \mathbf{u} e$. Then $c \mid e$.

Proof. Let $d:=a b c ; h \mid e, c$ and $g:=a b h$. Then $g \mid e$ and $g u h ;$ cf. 2.3 and 2.7. We have $g, h \mid e, g d=h c$, and $g \mathbf{u} h$. Hence $e=g d=h c \mid g, h, c, d$.

A similar argument shows

2.10'. Suppose that $\left(\mathrm{U}^{\prime \prime}\right)$ holds. Let $a b=c d$ and $q \mid c, d$ and $a \mathbf{u} b$. Then $q \mid a, b$. In particular, if $a b=c d$ and $a \mathbf{u} b$ and $c \mathbf{u} d$ then $q \mid a, b, c, d$ for some $q$.

2.11. Suppose $a|c, d ; p| b, c ; q \mid b, d ; b \mathbf{u} c, d$ and $p \mathbf{u} q$. Then $a \rightsquigarrow b$.

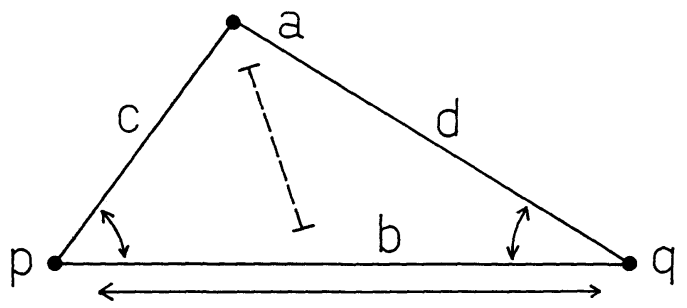


Proof. The assumptions yield $c \hookleftarrow q$, hence $a \mathbf{u} q$. Furthermore, $a \mid d$; $d|q ; q| b$ and $d \mathbf{u} b$. Thus $a \longmapsto b$.

2.12. Let $a, b \in S$. Then $a-b \Leftrightarrow c \mathbf{u} b$ for every $c \mid a$.

Proof. " $\Rightarrow$ " is (E1). " $\Leftarrow "$. Select any $c \mid a$. Then $c \mathbf{u} b$ by our assumption. Let $p \mid b, c$. Select $g \mid b$ such that $g \mathbf{u} p$; cf. (E2). Then $c \curvearrowleft g$, hence $a \mathbf{u} g$. Let $d \mid a, g$. Our assumption yields $d \mathbf{u} b$. Now $b \longmapsto a$ by definition.

Without success we made attempts to avoid (U) in our system of axioms, since an analytic model may fail to satisfy (U). Observe that (U) is valid in the particular case that $a \longmapsto b$ or $c \mid a, b$ for some $c$ holds for every pair $a, b$; cf. (E1) and 2.2(c).

3. The elliptic plane and the pseudo-plane. Let again $(G, S)$ satisfy the basic assumption and (E1), (E2), (E3), (U').

In Part I we introduced the group of motions $(\mathscr{G}, \mathscr{S})$ of an elliptic plane over a ring. $(\mathscr{G}, \mathscr{S})$ satisfies our system of axioms (except perhaps (U)). Every pair consisting of a point $R a$ and its polar line $R a^{*}$ corresponds to a reflection $\sigma_{R a}$. With this identification, incidence and orthogonality of the elliptic plane both correspond to the relation "p" on $\mathscr{S}$; cf. I.15. Hence, in order to reconstruct the elliptic plane, we must assign both a point and a line to each element of $\mathscr{S}$. Having this in mind we perform the following construction.

Select a bijection ^ ${ }^{\wedge}$ of $S$ onto a set $\mathscr{P}=\hat{S}$ such that $\mathscr{P} \cap G=\varnothing$. This bijection will be maintained throughout the rest of this article. Also for the rest of this article we fix an element $u \in S$. Let $\mathscr{L}:=S, \mathscr{L}^{\prime}:=\{g$ $\in \mathscr{L}: g \mathbf{u} u\}$ and $\mathscr{P}^{\prime}:=\{\hat{a} \in \mathscr{P}: a \longmapsto u\}$.

We regard $\mathscr{P}$ as a set of points, $\mathscr{L}$ as a set of lines. A point $\hat{a}$ is incident with a line $g$, abbreviated $\hat{a} \mid g$ or $g \mid \hat{a}$, if $a \mid g$ holds. Lines $g, h$ are called orthogonal, abbreviated $g \mid h$, if $g \mid h$ holds. The incidence structure $(\mathscr{P}, \mathscr{L}, \mid)$, together with this orthogonality, is called the elliptic plane assigned to $(G, S)$. The incidence structure $\left(\mathscr{P}^{\prime}, \mathscr{L}^{\prime}, \mid\right)$ is called the affine plane (with respect to $u)$. $\left(\mathscr{P}^{\prime}, \mathscr{L}^{\prime}, 1\right)$ is a locally complete substructure of $(\mathscr{P}, \mathscr{L}, \mid)$, i.e. if $\hat{a} \in \mathscr{P}^{\prime}$ and $g \in \mathscr{L}$ with $\hat{a} \mid g$ then $g \in \mathscr{L}^{\prime}$ (apply (E1)). $\hat{a}$ is called the polar point of the line $a$.

Let $\mathscr{D}:=\{x y: x, y \mid u\}$ and $\mathscr{D}^{*}:=\{x y: x, y \mid u$ and $x \mathbf{u} y\} . \mathscr{D}$ is an abelian subgroup of $G$; cf. (E3). For brevity, the elements of $\mathscr{D}$ will be called angles. $u$ is an element of $\mathscr{D}$ and is called the right angle. (More precisely, the elements of $\mathscr{D}$ will serve as quantities assigned to pairs of 
lines by an angle measure.) We define an angle measure

$$
\mathrm{w}: \mathscr{L}^{\prime} \times \mathscr{L}^{\prime} \rightarrow \mathscr{D}, \quad \mathrm{w}(g, h):=c d,
$$

where $c \mid g, u$ and $d \mid h, u .\left(\mathscr{P}^{\prime}, \mathscr{L}^{\prime}, \mid, \mathrm{w}\right)$ is called he pseudo-plane (of $(G, S)$ with respect to $u$ ). Two lines $g, h \in \mathscr{L}^{\prime}$ are called pseudo-parallel if $\mathrm{w}(g, h)=1$, and pseudo-orthogonal if $\mathrm{w}(g, h)=u$.

An angle $\alpha \in \mathscr{D}$ is called regular if for all $g, h \in \mathscr{L}^{\prime} \mathrm{w}(g, h)=\alpha$ implies that $g, h$ intersect in just one point of $\mathscr{P}^{\prime}$.

$$
\begin{aligned}
& \text { 3.1. } \mathrm{w}(g, h) \mathrm{w}(h, j)=\mathrm{w}(g, j) \text { and } \mathrm{w}(g, h)=\mathrm{w}(h, g)^{-1} \text { for all } g, h, j \\
& \in \mathscr{L}^{\prime} \text {. }
\end{aligned}
$$

3.2. Given $\hat{a} \in \mathscr{P}^{\prime}, g \in \mathscr{L}^{\prime}$ and $\alpha \in \mathscr{D}$ there is exactly one $h \in \mathscr{L}^{\prime}$ such that $\hat{a} \mid h$ with $\mathrm{w}(g, h)=\alpha$.

Proof. Let $c \in S$ with $c \mid g, u$, and $d:=c \alpha$. $a \smile u$ implies $a \mathbf{u} d$. Hence $h \mid a, d$ for exactly one $h \in S$. Furthermore, $h \in \mathscr{L}^{\prime}$.

\section{3. $\mathscr{D}^{*}$ is the set of regular angles.}

Proof. Let $g, h \in \mathscr{L}^{\prime}$ and $\alpha:=\mathrm{w}(g, h) \in \mathscr{D}^{*}$, hence $c \mathbf{u} d$ (by 2.7), where $c \mid g, u$ and $d \mid h, u$. Then $g \mathbf{u} h$ by 2.8 , i.e. there is exactly one $a \in S$ with $a \mid g, h$. We have $h|d ; d| u ; u \mid c$ and $h \mathbf{u} u$ and $d \mathbf{u} c$, hence $h \longmapsto c$ and $a \mathbf{u} c$. Furthermore, $a|g ; g| c ; c \mid u$ and $g \mathbf{u} u$. Therefore, $a \curvearrowleft u$, i.e. $\hat{a} \in \mathscr{P}^{\prime}$. Conversely, let us assume $\alpha \in \mathscr{D} \backslash \mathscr{D}^{*}$. We claim that $\alpha$ is not a regular angle. Select $c, d \in S$ with $c, d \mid u$ and $\alpha=c d$. Then $c \mathbf{u} d$ is not true, and 2.6 produces an element $a \in S$ with $a \neq u$ and $a \mid c, d$ and $a \longmapsto u$. Thus, $c, d \mid \hat{u}, \hat{a}$, i.e. the lines $c, d \in \mathscr{L}^{\prime}$ do not intersect uniquely in the pseudoplane and satisfy $\mathrm{w}(c, d)=\alpha$.

3.4. Let $g, h \in \mathscr{L}^{\prime}$ and $\hat{a} \in \mathscr{P}^{\prime}$ with $\hat{a} \mid g, h$. If $g \mathbf{u} h$ then $\mathrm{w}(g, h) \in \mathscr{D}^{*}$. The assertion follows immediately from 2.9.

REMARK. In 3.4 we were not able to replace the assumption $g \mathbf{u} h$ by the property that $\hat{a}$ is the only common point of $g$ and $h$ in the pseudo-plane.

4. Products of two elements of $S$. From now on we assume that $(G, S)$ satisfies (E1), (E2), (E3) and (U) of $§ 2$.

4.1. Let $a b=c d$ and $a \mathbf{u} b$. Then $c \mathbf{u} d$ and $e \mid a, b, c, d$ for some e. In particular, $a b c \in S$ and $a, b \mid e$ and $a \mathbf{u} b$ imply $c \mid e$. 
Proof. Let $e \mid a, b$. Select $f$ such that $f \mid c$ and $f \mathbf{u} e$; cf. (U). Let $r \mid e, f$ and $s:=r a b$. Then $s \mid e$ and $r u s ;$ cf. 2.3, 2.7. Select $q$ such that $q \mid f$ and $q \mathbf{u} d$; cf. (U). Then $q, e, r \mid f$, hence $m:=q c r=q c a b s=q d s \in S$ and $m \mid f$.

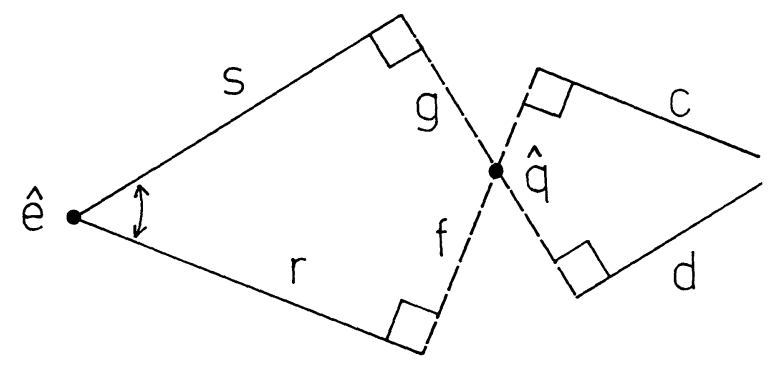

$s \mathbf{u} r$ and $e \mathbf{u} f$ yield $s \rightsquigarrow f$. In particular, $m \mathbf{u} s$. Also, $m s=q d$ and $q \mathbf{u} d$. Hence, $g \mid m, s, q, d$ for some $g$; cf. 2.10'. From $s \longmapsto f$ and $g \mid s$ follows $g \mathbf{u} f$. Together with $q \mathbf{u} d$ follows $c \mathbf{u} d$; cf. 2.8. Thus, $a b=c d$ and $a \mathbf{u} b$ and $c \mathbf{u} d$. $2.10^{\prime}$ yields immediately the assertion.

REMARK. 4.1 subsumes the statements 2.5 and 2.10 .

Due to 4.1 the following definition makes sense.

Definition. Let $\mathscr{E}:=\{a b: a \mathbf{u} b\}$. To every $a b \in \mathscr{E}$ corresponds a unique $c$ with $c \mid a, b$, called the support of $a b$. Let $\mathscr{F}:=\{g h q: g \mathbf{u} h$ and $\operatorname{support}(g h) \longmapsto q\} . \mathscr{F}_{0}:=\{p q: p \longmapsto q\}$.

The following remarks are obvious.

$a \mathbf{u} b$ if and only if $a b \in \mathscr{E}$, for any pair $a, b ;$ cf. 4.1. $\mathscr{E}, \mathscr{F}$ and $\mathscr{F}_{0}$ are invariant under inner automorphisms of $G$. Furthermore, $\mathscr{F}_{0} \subseteq \mathscr{F}$. If $\alpha \in \mathscr{E}, \mathscr{F}$ or $\mathscr{F}_{0}$, then $\alpha^{-1} \in \mathscr{E}, \mathscr{F}, \mathscr{F}_{0}$, respectively.

For arbitrary subsets $\mathscr{A}, \mathscr{B} \subseteq \mathrm{G}$ let $\mathscr{A} \mathscr{B}:=\{\alpha \beta: \alpha \in \mathscr{A}, \beta \in \mathscr{B}\}$.

4.2. $\mathscr{E}=S \mathscr{F}=\mathscr{F} S=S \mathscr{F}_{0}=\mathscr{F}_{0} S$. Furthermore, support $(x \mu) \longmapsto x$ for every $\mu \in \mathscr{F}$ and every $x \in S$.

Proof. If $\mu \in \mathscr{F}$ then $S \mathscr{F} \ni a \mu=\mu^{a} \cdot a \in \mathscr{F} S$ for any $a$. Thus the second and the last equality are clear. Also $\mathscr{F} S \supseteq \mathscr{F}_{0} S$. So it suffices to prove

(1) $\mathscr{F}_{0} S \supseteq \mathscr{E}$ and

(2) $\mathscr{E} \supseteq S \mathscr{F}$.

Proof of (1). Let $a b \in \mathscr{E}$. Then $a c \backsim b$, where $c \mid a, b$; cf. 2.2. Hence $a b=c(a c) b \in S \mathscr{F}_{0}$. 
Proof of (2). Let $g, h \mid p$ and $g \mathbf{u} h$ and $p \backsim q$. Let $x \in S$. We claim $x g h q \in \mathscr{E}$, and $y \longrightarrow x$, where $y:=\operatorname{support}(x g h q)$. Select $f \mid x$ with $f \mathbf{u} p ;$ cf. (U). Applying (E3) one can assume $g \mid f . p \curvearrowleft q$ yields $h \mathbf{u} q$. Let $r \mid h, q$. From 2.8 we obtain $f \mathbf{u} r$. Let $s \mid f, r$. Then $t:=x g s \in S$ and $t \mid f$. Also $v:=s h q \in S$ and $v \mid r$. We have $r|v, s ; f| t, s ; v \mathbf{u} s$ (namely $v s=q h \in \mathscr{E}$ since $q \longmapsto p$ ) and $f \mathbf{u} r$. Hence $t v \in \mathscr{E}$ by 2.8. Therefore, $x g h q=x g s s h=$ $t v \in \mathscr{E}$. From $g|p ; p| h ; h \mid r ; g \mathbf{u} h ; p \mathbf{u} r$ (since $p \curvearrowleft q$ ) follows $g \longmapsto r$. Thus $x t=g s \in \mathscr{E}$. We have observed $v \mathbf{u} s$ and $f \mathbf{u r} .2 .8$ yields $f \mathbf{u} y$. Together with $x|f ; f| t ; t \mid y$ follows $x \mapsto y$.

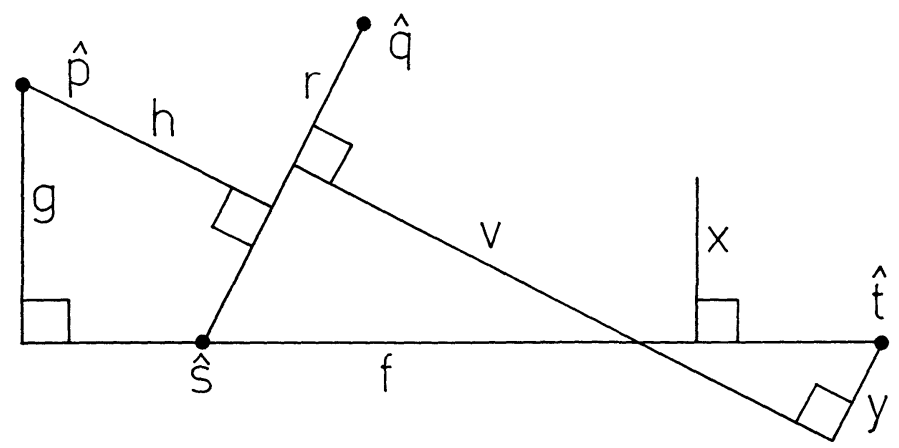

4.3. Let $a b=c d$. If $a \longmapsto b$ then $c \rightsquigarrow d$. In other words: $c d \in \mathscr{F}_{0}$ if and only if $c \hookleftarrow d$, for any pair $c, d$.

Proof. Let $g \mid c$. Then $g d=(g c) a b \in S \mathscr{F}_{0}=\mathscr{E}$; cf. 4.2. 2.12 yields the assertion.

\section{4. $\mathscr{F}_{0}=S S \cap \mathscr{F}$.}

Proof. Let $a b \in \mathscr{F}$. Let $c \mid a$. Then $c b=c a \cdot a b \in S \mathscr{F}=\mathscr{E}$, hence $c \mathbf{u} b$; cf. the remarks preceding 4.2. 2.12 yields the assertion.

4.5. $\mathscr{F}=\{\gamma \in G: \gamma x \in \mathscr{E}$ for all $x\}=\{\gamma \in G: x \gamma \in \mathscr{E}$ for all $x\}$

Proof. " $\subseteq$ " is an assertion of 4.2. Let $\gamma$ be an element of the right-hand set. Select an arbitrary $q$. Then $\gamma q=g h \in \mathscr{E}$. Let $p \mid g, h$. We want to prove $p \multimap q$. By 2.12 it is enough to verify $x q \in \mathscr{E}$ for any $x \mid p$. So let $x \mid p$. We can assume $h=x$; cf. (E3). Then $x q=g \gamma=(\gamma g)^{g} \in \mathscr{E}$ by our assumption.

REMARK. We shall prove a "representation theorem" for $\mathscr{F}$; cf. 6.4. This theorem is the synthetic counterpart of I.17. 
5. $G=\mathscr{E} S$. We continue to assume that $(G, S)$ satisfies (E1), (E2), (E3) and (U).

\section{1. $\mathscr{E} \mathscr{E} \subseteq S \mathscr{E}=\mathscr{E} S$.}

Proof. Let $\alpha, \beta \in \mathscr{E}$ and $a:=\operatorname{support}(\alpha), b:=\operatorname{support}(\beta)$. Due to (U) we can assume $\alpha=g h, \beta=r s, h \mid r$ and $h \mathbf{u} b$. Then $\alpha \beta=g(h r) s$ and $b \longmapsto h r$. Hence $s \mathbf{u} h r$, and $\alpha \beta \in S \mathscr{E}$.

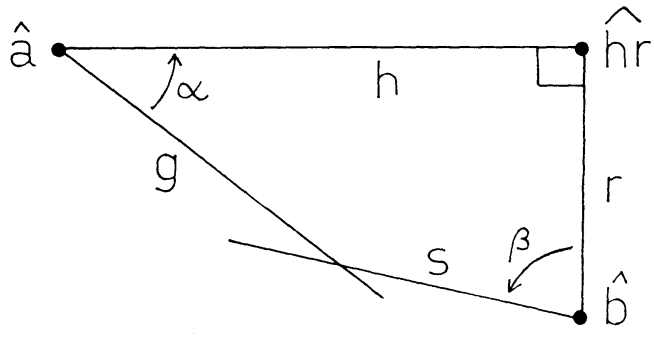

\section{2. $S S S \subseteq \mathscr{E} \mathscr{E} \subseteq \mathscr{E} S$}

Proof. Given $a, b, c$. Select $g, h$ such that $g|c ; g \mathbf{u} b ; h| b, g$; cf. (U). From 2.4(i) and 2.2 we obtain $v$ such that $v \mid h$ and $v \longmapsto b, g$. Then $v \mathbf{u} g h$ and $v \mathbf{u} c$ since $v \longmapsto g$ and $g \mid c$. Thus,

$$
a b c=(a b v)(v c) \in S \mathscr{F} \mathscr{E} \subseteq \mathscr{E} \mathscr{E} ;
$$

cf. 4.2. 5.1 finally yields the conclusion $a b c \in \mathscr{E} S$.

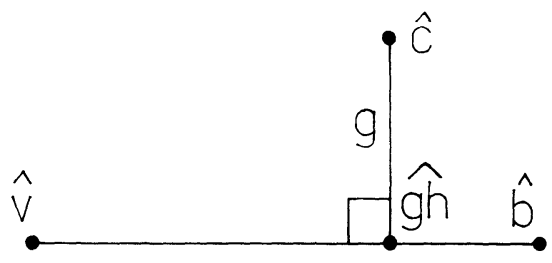

5.3. $G=S S S S=\mathscr{E} S S$.

Proof. 5.1 and 5.2 yield

$S S S S S \subseteq \mathscr{E} S \cdot S S \subseteq \mathscr{E} \mathscr{E} S \subseteq \mathscr{E} S S \subseteq S S S S \subseteq S S S S S$

This proves the assertion.

5.4. $G=\mathscr{E} S=S \mathscr{E}$ 
Proof. Let $\alpha \in G$. We can write $\alpha=\beta c d$, where $\beta \in \mathscr{E}$; cf. 5.3. Let $s:=\operatorname{support}(\beta)$. Select $t$ such that $t \mid c$ and $t \mathbf{u} s$; cf. (U). Then $\beta=a b$ for some $a, b$ with $b \mid s, t$ and $a \longmapsto t$. Select $q$ such that $q \mid d$ and $q \mathbf{u} t$. Using (E3) we obtain $b c=b^{\prime} c^{\prime}$, where $b^{\prime}, c^{\prime} \mid t$ and $c^{\prime} \mid q$. qu $t$ implies $q \longmapsto t c^{\prime}$, and $a \curvearrowleft t$ implies $a \mathbf{u} b^{\prime} t$. Thus,

$$
\alpha=a b c d=a b^{\prime} c^{\prime} d=a\left(b^{\prime} t\right)\left(t c^{\prime}\right) d \in \mathscr{E} \mathscr{E} \subseteq S \mathscr{E} ;
$$

cf. 5.1 .

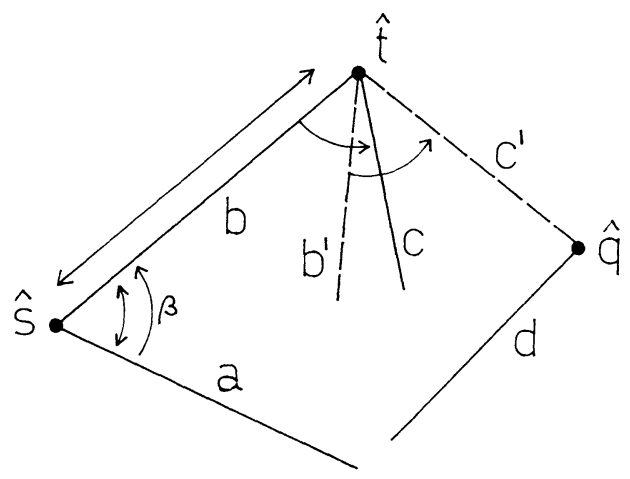

5.5. If (M) holds then $C_{G}=\left\{\alpha \in G: x^{\alpha}=x\right.$ for every $\left.x\right\}=1$.

Proof. Let $\alpha \in C_{G}$. We have $\alpha=\beta c$, where $\beta \in \mathscr{E}$; cf. 5.4. Select $t$ such that $t \mid c$ and $t \mathbf{u} s:=\operatorname{support}(\beta)$; $\operatorname{cf}$. (U). Then $\beta=a b$ for some $a, b$ with $b \mid s, t . t^{\alpha}=t$ yields $t^{a}=t$, hence $t=a$ by $(\mathrm{M})$, since $t \rightsquigarrow a$. Let $d:=a b$. Then $d \in S$ and $c, d \mid a$ and $\alpha=d c$. Select $e$ such that $e \mid d$ and $e \mathbf{u} a$ and $e \curvearrowleft a$; cf. (E2). Then $e \mathbf{u} c$. Let $s \mid e, c$. We have $e \curvearrowleft c s$ and $e=e^{\alpha}=e^{c}=e^{c s}$, hence $e=c s$; cf. (M). Finally, from $a, e \mid d, c$ and $a \mathbf{u} e$ follows $d=c$, i.e. $\alpha=1$.

6. A class of automorphisms of $(\mathscr{P}, \mathscr{L}, \mid)$.

6.1. Definition. To every $\gamma \in \mathscr{F}$ (cf. $\S 4.1$ ) we define a mapping $T_{\gamma}$ : $S \rightarrow S, x \mapsto \operatorname{support}(x \gamma)$; cf. $\S 4.1$. We collect some properties of $T_{\gamma}$.

6.2. Let $\gamma \in \mathscr{F}$.

(a) $x \longmapsto x T_{\gamma}$ for every $x$.

(b) $T_{\gamma}$ is a bijection.

(c) $a\left|b \Leftrightarrow a T_{\gamma}\right| b T_{\gamma^{-1}}^{-1}$ for all $a, b$. In other words: $a\left|b T_{\gamma} \Leftrightarrow a T_{\gamma^{-1}}\right| b$.

(d) $a \mathbf{u} b \Leftrightarrow a T_{\gamma} \mathbf{u} b T_{\gamma}$ for all $a, b$. 
Proof. (a) follows from 4.2.

(b) Given $y \in S$ and $\gamma=g h q$ with $p \mid g, h$ and $g \mathbf{u} h$ and $p \multimap q$. We want to show $y=\operatorname{support}(x \gamma)$ for just one $x$. Consider the figure of 4.2. From (U) we obtain $r, v$ such that $v \mid y$ and $v \mathbf{u} q$ and $r \mid q, v$. Then $p \mathbf{u} r$ since $p \hookleftarrow q$. Using (E3) we may therefore assume $h \mid p, r$. Let $s:=h q v$. Then $s \mid r$ and $s h=v q \in \mathscr{E}$. From $s \mathbf{u} h$ and $r \mathbf{u} p$ we obtain $s \rightsquigarrow p$, hence $s \mathbf{u} g$. Let $f \mid s, g . g \mathbf{u} h$ and $p \mathbf{u} r$ yield $f \mathbf{u} r$; cf. 2.8. Together with $s \mathbf{u} v$ follows $f \backsim v$, hence $f \mathbf{u} y$. Let $t \mid f, y$. Now $x:=t s g$ satisfies $x \gamma=t v$ and $\operatorname{support}(x \gamma)=\operatorname{support}(t v)=y$. Let us assume support $\left(x^{\prime} \gamma\right)=y$ for some $x^{\prime}$. Perform the construction in the proof of (2) in 4.2 with $g^{\prime}, h^{\prime}, r^{\prime}, \ldots$ instead of $g, h, r, \ldots$ Then $x^{\prime} \gamma=t^{\prime} v^{\prime}$ and $x \gamma=t v$, where $t^{\prime}, v^{\prime}, t, v \mid y=y^{\prime}$. Let $t^{\prime \prime}:=t^{\prime} v^{\prime} v$. Then $t^{\prime \prime} \mid y$ and $t^{\prime \prime} x^{\prime}=t x \in \mathscr{E}$ as $y \longrightarrow x$ and $t \mid y$. Therefore $t, t^{\prime \prime} \mid y, f$. Furthermore, $y \mathbf{u} f$ as $y \longmapsto x$. Hence $t=t^{\prime \prime}$ and $x=x^{\prime}$.

(c) Let $x:=a T_{\gamma}$ and $y:=b T_{\gamma^{-1}}^{-1}$; i.e. $x=\operatorname{support}(a \gamma)$ and $b=$ support $\left(y \gamma^{-1}\right)$. The following statements are equivalent. $a \mid b ; y \gamma^{-1} a \in S$; $a \gamma y \in S ; y \mid x$.

(d) follows immediately from (b) and (c).

6.3. For every $\gamma \in \mathscr{F}$ the mapping $\mathscr{L} \rightarrow \mathscr{L}, g \mapsto g T_{\gamma}$ induces an automorphism of $(\mathscr{P}, \mathscr{L}, \mid)$.

Proof. Define a mapping $\mathscr{P} \rightarrow \mathscr{P}, \hat{x} \mapsto \hat{y}$, where $y=x T_{\gamma^{-1}}^{-1}$ The pair, consisting of the two mappings, is an automorphism of $(\mathscr{P}, \mathscr{L}, 1)$; cf. 6.2(b) and (c).

6.4. Let $\gamma \in \mathscr{F}$ and $p \in S$.

(a) Let $g h q=\gamma=g^{\prime} h^{\prime} q^{\prime}$, where $g, h, g^{\prime}, h^{\prime} \mid p$. Then $g h=g^{\prime} h^{\prime} \in \mathscr{E}$ and $q=q^{\prime} \longmapsto p$.

(b) $\gamma=\beta q$ for some $\beta \in \mathscr{E}$ and $q$ such that $\operatorname{support}(\beta)=p$ and $p \varpi q$.

Proof. (a) We have $g h=\gamma q \in \mathscr{F} S=\mathscr{E}$; cf. 4.5 .

$$
\begin{aligned}
q T_{\gamma^{-1}} & =\operatorname{support}\left(q \gamma^{-1}\right)=\operatorname{support}(h g)=p=\operatorname{support}\left(h^{\prime} g^{\prime}\right) \\
& =\operatorname{support}\left(q^{\prime} \gamma^{-1}\right)=q^{\prime} T_{\gamma^{-1}} .
\end{aligned}
$$

Hence $q=q^{\prime} \longmapsto p$ by 6.2(a) and (b).

(b) Let $\delta:=\gamma^{-1}$. The mapping $T_{\delta}$ is surjective; cf. 6.2(b). Hence $q T_{\delta}=p$ for some $q$; i.e. $\operatorname{support}(\beta)=p$, where $\beta=q \delta$. Furthermore, $p \longmapsto q$ by $6.2(\mathrm{a})$. 
7. Pseudo-semirotations. We refer to the notions and notations introduced in $\S 3$. In particular, $u \in S$ is a fixed element, and the point set of the pseudo-plane is $\mathscr{P}^{\prime}=\{\hat{a}: a \longmapsto u\}$; the set of lines is $\mathscr{L}^{\prime}=\{g$ : $g \mathbf{u} u\}$.

Our next aim is to show that the pseudo-plane is a generalized semi-rotation plane in the sense of [8].

Let $\alpha \in \mathscr{D}$ and $\hat{y} \in \mathscr{P}^{\prime}$. The pseudo-semi-rotation $H=H_{\hat{y}, \alpha}$ assigned to the center $\hat{y}$ and the angle $\alpha$ is a mapping of $\mathscr{L}^{\prime}$ into $\mathscr{L}^{\prime}$, where $x H$ is the line passing through the foot of the pseudo-orthogonal of $x$ through $\hat{y}$

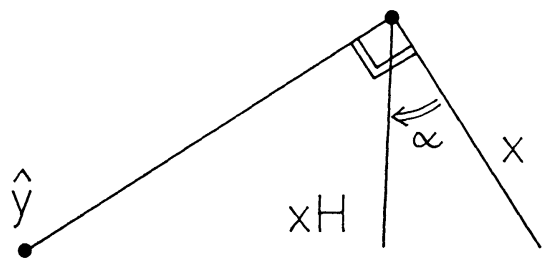

such that $\mathrm{w}(x, x H)=\alpha$. This definition yields immediately:

7.1. $\mathrm{w}(x, x H)=\alpha$ for every $x \in \mathscr{L}^{\prime}$ and $H=H_{\hat{y}, \alpha}$. In particular, $\mathrm{w}(x, z)=\mathrm{w}(x H, z H)$ for all $x, z \in \mathscr{L}^{\prime}$.

7.2. Let $a_{1}, a_{2}, a_{3} \in \mathscr{L}^{\prime}$ and $\hat{x} \in \mathscr{P}^{\prime}$ with $a_{1}, a_{2}, a_{3} \mid \hat{x}$. Let us assume that $\mathrm{w}\left(a_{1}, a_{2}\right) \in \mathscr{D}^{*}$. Then every pseudo-semi-rotation $H$ satisfies $a_{3} H \mid \hat{z}$, where $\hat{z} \in \mathscr{P}^{\prime}$ is the unique point with $\hat{z} \mid a_{1} H, a_{2} H$.

Proof. Let $H=H_{\hat{y}, \alpha}$. For $h \in \mathscr{L}^{\prime}$ let $h^{\prime} \in S$ be the element with the property $h^{\prime} \mid u, h$. For $i=1,2,3$ let $c_{i}=a_{i} H$ and let $b_{i} \in \mathscr{L}^{\prime}$ denote the pseudo-orthogonal of $a_{i}$ through $\hat{y}$. Let $\hat{d}_{i}$ denote the foot. With these

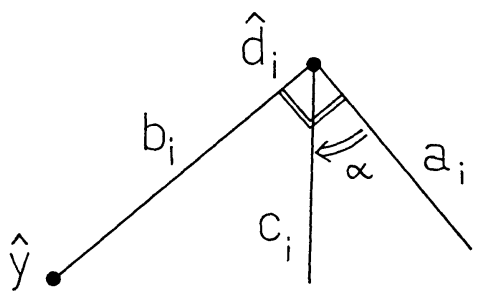


notations the definition of $H$ implies for $i=1,2,3$ :

$$
\begin{aligned}
& a_{i}^{\prime} b_{i}^{\prime}=u \quad \text { and } \quad a_{i}^{\prime} c_{i}^{\prime}=\alpha \\
& x_{i}:=x a_{i}^{\prime} d_{i} \in S \quad \text { as } x, a_{i}^{\prime}, d_{i} \mid a_{i} \quad \text { (cf. (E3)), } \\
& y_{i}:=y b_{i}^{\prime} d_{i} \in S \quad \text { as } y, b_{i}^{\prime}, d_{i} \mid b_{i},
\end{aligned}
$$

and for $i=1,2$

$$
z_{i}:=z c_{i}^{\prime} d_{i} \in S \quad \text { as } z, c_{i}^{\prime}, d_{i} \mid c_{i} .
$$

Furthermore, $a_{1}^{\prime} a_{2}^{\prime} \in \mathscr{D}^{*}$.

An elementary calculation yields

$$
x_{1} x_{2}=y_{1} y_{2}=z_{1} z_{2} \text { and } x_{2} x_{3}=y_{2} y_{3}=z_{2} z_{3} \text {, }
$$

where $z_{3}:=z c_{3}^{\prime} d_{3}$ but $z_{3} \in S$ is still unknown.

We have $a_{2}\left|d_{2}, x_{2} ; b_{2}\right| d_{2}, y_{2} ; a_{2} b_{2} \in \mathscr{E}\left(\right.$ since $\left.\mathrm{w}\left(a_{2}, b_{2}\right)=u \in \mathscr{D}^{*}\right)$ and $x_{2} d_{2}=x a_{2}^{\prime} \in \mathscr{E}$ as $a_{2}^{\prime} \mid u$ and $u \longrightarrow x .2 .8$ implies

$$
x_{2} y_{2} \in \mathscr{E} \text {. }
$$

We apply 2.8 once more, using the relations $a_{1}\left|x, x_{1} ; a_{2}\right| x, x_{2} ; x x_{1}=$ $a_{1}^{\prime} d_{1} \in \mathscr{E}\left(\right.$ since $a_{1}^{\prime} \mid u$ and $\left.u \backsim d_{1}\right) ; a_{1} a_{2} \in \mathscr{E}\left(\right.$ since $\left.\mathrm{w}\left(a_{1}, a_{2}\right) \in \mathscr{D}^{*}\right)$. Thus

$$
x_{1} x_{2} \in \mathscr{E} \text {. }
$$

Let $e:=\operatorname{support}\left(x_{2} y_{2}\right)$. (3) implies $x_{2} y_{2} x_{1} \in S$ and $x_{2} y_{2} y_{3} \in S$. (4) and 5.1 yield $x_{1}, y_{3} \mid e$. Therefore, support $\left(x_{1} x_{2}\right)=e$. Since $x_{1} x_{2} z_{2} \in S$ we obtain $z_{2} \mid e$; cf. 4.1. A simple calculation involving only (1) and (2) yields $z_{3}=z_{2} y_{2} y_{3}$. We have proved $z_{2}, y_{2}, y_{3} \mid e$. Hence $z_{3} \in S$. This stands for $z c_{3}^{\prime} d_{3} \in S$. Moreover, $c_{3}^{\prime} d_{3} \in \mathscr{E}$ and $c_{3}=\operatorname{support}\left(c_{3}^{\prime} d_{3}\right)$ since $c_{3}^{\prime} \mid u$ and $u \longmapsto d_{3} .4 .1$ implies $z \mid c_{3}$.

7.3. The following property holds in the pseudo-plane. $(\Delta)$ There are regular angles $\alpha, \beta, \gamma \in \mathscr{D}^{*}$ such that $\alpha \beta=\gamma$.

Proof. Select $g, h, j$ with $g, h, j \mid u$ and $g \mid h$ and $j \mathbf{u} g, h$. This choice is possible by $2.4(\mathrm{i})$. Let $\alpha:=g j, \beta:=j h, \gamma:=u . \alpha, \beta, \gamma$ fulfill the desired property; cf. 3.3 .

7.4. To any $x$ there exist $a_{1}, a_{2}$ such that $a_{1}, a_{2} \mid x ; a_{1} \mathbf{u} a_{2} ; a_{1}, a_{2} \mathbf{u} u$; and $c \mid a_{2}$ with $c \longmapsto u, a_{1}$ for some $c$.

Proof. From (U) we get $a_{1} \mid x$ with $a_{1} \mathbf{u} u$. Let $r \mid u, a_{1}$. As $a_{1} r \sqcup u$ (cf. 2.2) there is $c \mid r$ with $c-u$ and $c \mathbf{u} a_{1} r$; cf. 2.4(ii). From $c-a_{1}$ and $a_{1} \mid x$ 
follows $c \mathbf{u} x$. Let $a_{2} \mid x, c$. We conclude $a_{1} \mathbf{u} a_{2}$ and $a_{2} \mathbf{u} u$; cf. (E1).

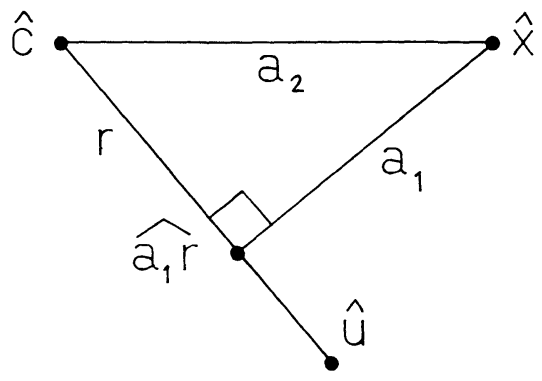

7.2 and 7.4 assert that every pseudo-semirotation $H=H_{\hat{y}, \alpha}$ induces a (unique) mapping $\mathscr{P}^{\prime} \rightarrow \mathscr{P}^{\prime}$ such that $g \mid \hat{a}$ implies $g H \mid \hat{a} H$ for each $g \in \mathscr{L}^{\prime}$ and $\hat{a} \in \mathscr{P}^{\prime}$. Hence we shall regard a pseudo-semi-rotation as a mapping of $\mathscr{L}^{\prime}$ and $\mathscr{P}^{\prime}$.

It is easy to check that $H_{\hat{y}, \alpha}$ is injective if and only if $u \alpha \in \mathscr{D}^{*}$, i.e. $\alpha \in \mathscr{F}$. Then $H=H_{\hat{y}, \alpha}$ is already a bijection (of $\mathscr{L}^{\prime}$ and $\mathscr{P}^{\prime}$ ) with $g|\hat{a} \Leftrightarrow g H| \hat{a} H$.

7.5. Let $\alpha \in \mathscr{D} \cap \mathscr{F}$ and $\hat{y} \in \mathscr{P}^{\prime}$. Then $g H_{\hat{y}, \alpha}=g T_{y u}^{-1} T_{y u \alpha}$ for any $g \in \mathscr{L}^{\prime}$. Each bijective pseudo-semirotation can be uniquely extended to an automorphism of $(\mathscr{P}, \mathscr{L}, \mid)$ (the elliptic plane where the orthogonality is not taken into account).

Proof. $y u \in \mathscr{F}$ since $y \sqsupset u$. Write $\alpha=b c$ with $b, c \mid u$ and $u=a b$. Then $u \alpha \in \mathscr{E}$ by 4.2 and $u \alpha=a c$, so $a \mathbf{u c}$. Further, $y u \alpha=(a c y)^{y} \in \mathscr{F}$, since $\operatorname{support}(a c)=u \longmapsto y$. Let $H:=H_{\hat{y}, \alpha}, g \in \mathscr{L}^{\prime}$ and $h:=g H$. Take $g^{\prime} \mid u, g$ and $h^{\prime} \mid u, h$. Then $\alpha=g^{\prime} h^{\prime}$. Let $c$ denote the pseudo-orthogonal of $g$ through $\hat{y}$ and let $\hat{f}$ be the foot. Then $y, g^{\prime} u, f \mid c$, hence $z:=f\left(g^{\prime} u\right) y \in$ $S$ and $z \mid c$. We have $z y u=f g^{\prime}$. Therefore $z T_{y u}=g$. Now $z y u \alpha=f g^{\prime} \alpha=f h^{\prime}$ yields $z T_{y u \alpha}=\operatorname{support}\left(f h^{\prime}\right)=h$. The second assertion follows from the first one together with 6.3 and 7.4.

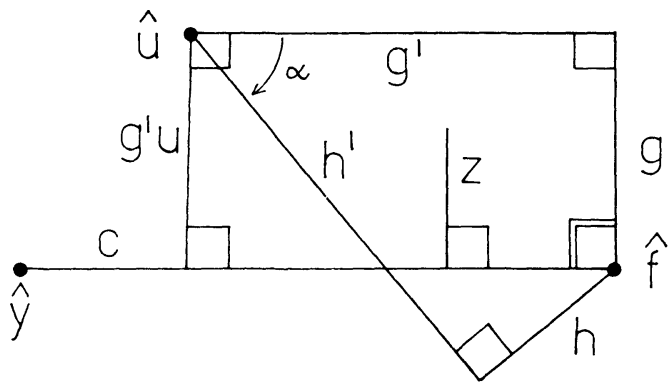


8. Transvections. Let $g, h \in \mathscr{L}^{\prime}$. We write $g(u) h$ if $x \mid g$ and $x-h$, or $x \mid h$ and $x \curvearrowleft g$ for some $\hat{x} \in \mathscr{P}^{\prime}$. Clearly, $g(u) h$ implies $g u h$.

We need this technical term in the proof of 9.1.

8.1. Let $g, h \in \mathscr{L}^{\prime}$ and $\hat{a} \in \mathscr{P}^{\prime}$ such that $a \mid g, h$ and $g \mathbf{u} h$. Then $g(\mathbb{u} h$.

Proof. Let $b \mid g, u$. Then $b \mathbf{u} a$. Select $c \mid g$ such that $c \mathbf{u} a, b$; cf. 2.4(i). Then $\hat{c} \in \mathscr{P}^{\prime}$ and $c \varpi h$.

8.2. Let $a \in S$. Then $g, h \mid a$ for some $g, h \in \mathscr{L}^{\prime}$ with $g(u) h$.

This is a reformulation of 7.4.

8.3. Let $g, h|u ; a| h ; a \mathbf{u} u ; g \mathbf{u} h$. Define $\mathscr{N}^{(i)}$ recursively:

$$
\begin{aligned}
& \mathscr{N}^{(0)}:=\{\hat{a}\} \cup\{\widehat{u g}\} \cup\left\{\hat{y} \in \mathscr{P}^{\prime}: y \mid g\right\} \cup\left\{x \in \mathscr{L}^{\prime}: x \mid u\right\}, \\
& \mathscr{N}^{(i+1)}:=\mathscr{N}^{(i)} \cup\left\{\hat{y} \in \mathscr{P} y \mid x_{1}, x_{2} \text { and } x_{1} @ x_{2} \text { for some } x_{1}, x_{2} \in \mathscr{N}^{(t)}\right\} \\
& \cup\left\{x \in \mathscr{L}^{\prime}: x \mid y_{1}, y_{2} \text { and } y_{1} \mathbf{u} y_{2} \text { for some } \hat{y}_{1}, \hat{y}_{2} \in \mathscr{N}^{(i)}\right\} .
\end{aligned}
$$

Then $\mathscr{N}^{(8)}=\mathscr{P} \cup \mathscr{L}^{\prime}$.

Proof. Let $c:=u g$. Select $b \mid g$ such that $b \hookrightarrow u$ and $b \mathbf{u} u$; cf. (E2). Then $c \mathbf{u} b, u$ and $\hat{b}, \hat{c} \in \mathcal{N}^{(0)}$. Then $a \mathbf{u} b$ since $a \backsim g$. Let $e \mid a, b$ and let $f$ denote the pseudo-parallel of $e$ through $\hat{u}$. Then $g \mathbf{u} e, f ; e \backsim u ; c \curvearrowleft e, f$.

$$
\{\hat{y} \in \mathscr{P}: y \mid e\} \subseteq \mathscr{N}^{(2)} \text {. }
$$

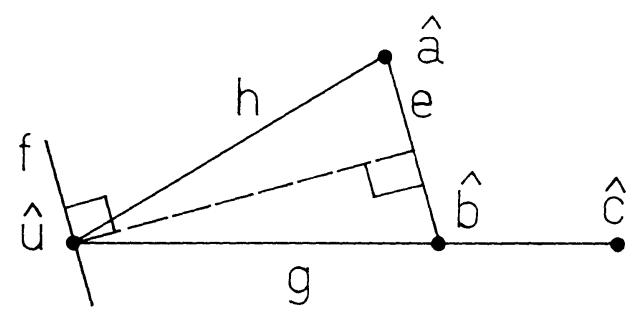

Proof. Let $y \mid e$. As $u \backsim e$ there is a unique $x \in \mathscr{L}^{\prime}$ with $x \mid \hat{u}, \hat{y}$. Then $x$ (1) $e$. From $e \in \mathscr{N}^{(1)}$ and $x \in \mathscr{N}^{(0)}$ it follows that $\hat{y} \in \mathscr{N}^{(2)}$.

$$
\{\hat{y} \in \mathscr{P}: y \mid f \text { and } y \varpi u\} \subseteq \mathscr{N}^{(4)} \text {. }
$$


Proof. Let $y \mid f$ and $y \longrightarrow u$. Then $x \mid y, c$ for a unique $x$. We have $c \longmapsto e$, hence $z \mid e, x$ and $z \mathbf{u} c$ for some $\hat{z} \in \mathscr{P}$. Then $\hat{z} \in \mathscr{N}^{(2)}$ by (1). Also, $\hat{c} \in \mathscr{N}^{(0)}$ and $z \mathbf{u} c$. Hence $x \in \mathscr{N}^{(3)} . c \longmapsto f$ implies $x \mathbf{u} f$. Select $w \mid f$ such that $w \curvearrowleft u$ and $w \mathbf{u} y$; cf. 2.4(ii). Then $\hat{w} \in \mathscr{P}^{\prime}$ and $w \curvearrowleft x$. Hence $f(\mathbb{U}) x$. Together with $f \in \mathscr{N}^{(0)}$ follows $\hat{y} \in \mathscr{N}^{(4)}$.

$$
\left\{x \in \mathscr{L}^{\prime}: y \mid f, x \text { and } z \mid e, x \text { for some } y, z \text { with } y \rightsquigarrow u\right\} \subseteq \mathscr{N}^{(5)} .
$$

Proof. From $y \mathbf{u} u f$ and $f \mathbf{u} e$ follows $y \hookleftarrow e$, hence $y \mathbf{u} z$. Together with (1) and (2) this yields $x \in \mathscr{N}^{(5)}$.

$$
\mathscr{P}^{\prime} \subseteq \mathscr{N}^{(6)}
$$

Proof. Let $y \multimap u$. Select $j \mid u$ with $j \mathbf{u} f$ and $j \multimap f$; cf. (E2). Let $m:=j u$. Then $m \mathbf{u} f$. Let $j^{\prime}$ and $m^{\prime}$ denote the pseudo-parallels of $j, m$, respectively, through the point $\hat{y}$. Then any two of the lines $e, j^{\prime}, m^{\prime}$, and also of the lines $f, j^{\prime}, m^{\prime}$, intersect uniquely in a point of the pseudo-plane. Therefore, $j^{\prime}, m^{\prime} \in \mathscr{N}^{(5)}$; cf. (3). Also, $j^{\prime} \mathbf{u} m^{\prime}$. Therefore $j^{\prime}(4) m^{\prime}$ by 8.1. Thus, $\hat{y} \in \mathscr{N}^{(6)}$.

Clearly, (4) implies $\mathscr{L}^{\prime} \subseteq \mathscr{N}^{(7)}$. Therefore $\mathscr{P} \subseteq \mathscr{N}^{(8)}$ by 8.2.

8.4. Definition. Let $\hat{z} \in \mathscr{P}$ and $g \in \mathscr{L}$ such that $g \mid z$. An automorphism $\tau$ of $(\mathscr{P}, \mathscr{L}, \mid)$ is called a transvection whose center is $\hat{z}$ and whose axis is $g$ if $\hat{x} \tau=\hat{x}$ and $y \tau=y$ for any point $\hat{x}$ of $g$ and any line $y$ through $\hat{z}$.

8.5. Let $z \mid g$ and $a, b, z \mid h$ such that $a, b \mathbf{u} z$ and hug. There is at most one transvection $\tau$ having axis $g$ and center $\hat{z}$ such that $\hat{a} \tau=\hat{b}$.

Proof. Suppose, $\tau_{1}$ and $\tau_{2}$ satisfy the above properties. We apply 8.3 with $z$ instead of $u$. The automorphism $\bar{\tau}:=\tau_{1} \tau_{2}^{-1}$ of $(\mathscr{P}, \mathscr{L}, \mid)$ fixes each point and each line of $\mathscr{N}^{(0)}$. 8.3 implies that $\bar{\tau}$ is the identity on the set $\mathscr{N}^{(8)} \supseteq \mathscr{P}$.

8.6. Let $z \mid g$ and $a, b, z \mid h$ such that $a, b \mathbf{u} z$ and hug. There is $a$ transvection $\tau$ of $(\mathscr{P}, \mathscr{L}, \mid)(c f .8 .4)$ whose center is $\hat{z}$ and whose axis is $g$ such that $\hat{a} \tau=\hat{b} . \tau$ is unique. Moreover, if $y \mathbf{u} g$ then $y \tau \mathbf{u} g$ for any line $y \in \mathscr{L}$; and if $x \longmapsto g$ then $x^{\prime} \mapsto g$, where $\hat{x} \tau=\widehat{x^{\prime}}$, for any point $\hat{x} \in \mathscr{P}$.

Proof. 8.5 states the uniqueness. 
In order to construct $\tau$ we may assume $g=u$, since our notion of the pseudo-plane may be introduced with respect to an arbitrary element $g \in S$. Then $\hat{a}, \hat{b} \in \mathscr{P}^{\prime}$ and $h \in \mathscr{L}^{\prime}$.

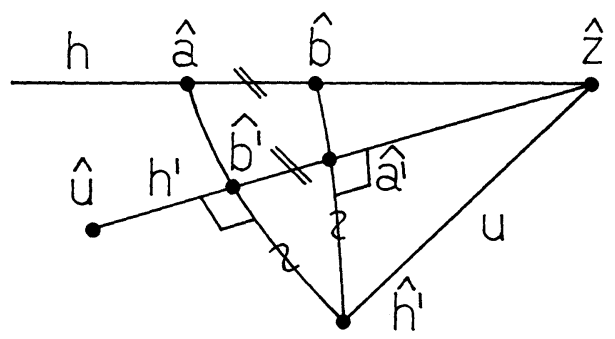

Secondly, we may assume $h \mid u$. Namely, let $h^{\prime}$ denote the pseudoparallel of $h$ through $\hat{u}$; let $\widehat{a^{\prime}}, \widehat{b^{\prime}}$ be the feet of the pseudo-orthogonals of $h^{\prime}$ through $\hat{a}, \hat{b}$, respectively. If $\tau$ is a transvection with center $\hat{z}$ and axis $u$ such that $\widehat{a^{\prime}} \tau=\widehat{b^{\prime}}$ then clearly $\tau$ will also fulfill $\hat{a} \tau=\hat{b}$. Let $m:=b h$. Select $v$ such that $v \mid m ; v-h ; v \mathbf{u} h$; cf. (E2). Then $v \mathbf{u} a, u$ and $v-u$. Thus, $\hat{v} \in \mathscr{P}^{\prime}$ and $r \mid a, v$ for some $r$ with $r \mathbf{u} h . \alpha:=\mathrm{w}(r, m) \in \mathscr{F}_{0} \cap \mathscr{D}$. Let $\tau:=T_{v u}^{-1} T_{v u \alpha} T_{b u \alpha}^{-1} T_{b u} ;$ cf. $\S 6$ and 7.5. We regard $\tau$ as a mapping $\mathscr{L} \rightarrow \mathscr{L} . \tau$

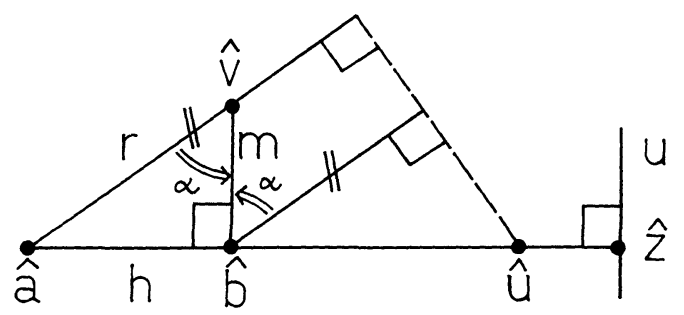

induces an automorphism of $(\mathscr{P}, \mathscr{L}, 1)$; cf. 6.3. Let $\tau$ also denote this automorphism. We contend that $\tau$ satisfies our requirements. 7.5 implies

(1) $H_{\hat{v}, \alpha} H_{\hat{b}, \alpha}^{-1}$ is the restriction of $\tau$ to the pseudo-plane $\left(\mathscr{P}^{\prime}, \mathscr{L}^{\prime}, \mid\right)$. From this we conclude

(2) $y \tau=y$ for any $y \in \mathscr{L}^{\prime}$ with $y \mid z$,

(3) $y$ pseudo-parallel $y \tau$ for any $y \in \mathscr{L}^{\prime}$,

(4) $\hat{a} \tau=\hat{b}$.

Since $u T_{v u}^{-1}=v, v T_{v u \alpha}=u, u T_{b u \alpha}^{-1}=b$ and $b T_{b u}=u$ one obtains

(5) $u \tau=u$.

The statements (3) and (5) imply

(6) $\hat{x} \tau=\hat{x}$ for each point $\hat{x}$ of the line $u$.

Finally, we want to prove

(7) $y \tau=y$ for each line $y$ through the point $\hat{z}$. 
(If $y$ is an affine line or if $y=u$ then this is clear; cf. (2) and (5). For an arbitrary $y$ however the arguments are not at all obvious.) Let $y \mid z$. We have $h=u z=b m$. Hence $m z=b u \in \mathscr{F}$ and $y \mathbf{u} m$. Let $e \mid y, m$ and $y^{\prime}:=y T_{b u}^{-1}$. The identity

$$
\operatorname{support}(e m b u)=\operatorname{support}(e z)
$$

together with $e, z \mid y$ yields $y^{\prime}=e m$. Furthermore, $y^{\prime}, b, v \mid m$. Hence, $y^{\prime \prime}:=y^{\prime} b v \in S$ and $y^{\prime \prime} \mid m$. Thus, by the above observation,

$$
\operatorname{support}\left(y^{\prime \prime} v u\right)=\operatorname{support}\left(y^{\prime} b v v u\right)=\operatorname{support}\left(y^{\prime} b u\right)=y \text {. }
$$

In other words, $y^{\prime \prime}=y T_{v u}^{-1}$. We have proved

$$
\begin{aligned}
y T_{v u}^{-1} T_{v u \alpha} & =y^{\prime \prime} T_{v u \alpha}=\operatorname{support}\left(y^{\prime \prime} v u \alpha\right)=\operatorname{support}\left(y^{\prime} b v v u \alpha\right) \\
& =\operatorname{support}\left(y^{\prime} b u \alpha\right)=y^{\prime} T_{b u \alpha}=y T_{b u}^{-1} T_{b u \alpha} .
\end{aligned}
$$

This means that $y \tau=y$.

The additional assertion of 8.6 is clear from (1).

9. Coordinates. If $R$ is a commutative ring with 1 then $\Pi(R)=$ $(\mathscr{P}(R), \mathscr{L}(R), \mathrm{I})$ will denote the projective plane over $R, \Pi^{\prime}(R)=$ $\left(\mathscr{P}^{\prime}(R), \mathscr{L}^{\prime}(R), \mathrm{I}\right)$ will denote the affine plane over $R$, viewed as a substructure of $\Pi(R)$, where $R[0,0,1]$ is the line of infinity.

9.1. There is a commutative ring $R$ with 1 and an embedding $\xi$ of the pseudo-plane $\left(\mathscr{P}^{\prime}, \mathscr{L}^{\prime}, \mid\right)$ (viewed as an incidence-structure) into the affine plane $\Pi^{\prime}(R)$, and an element $k \in R^{*}$ (group of units) with the following properties.

(i) $\mathscr{P}^{\prime} \xi=\mathscr{P}^{\prime}(R) . R[0,1,0], R[1,0,0] \in \mathscr{L}^{\prime} \xi, \hat{u} \xi=R(0,0,1)$.

(ii) $D_{0}:=\left\{R(\alpha, \beta): \alpha, \beta \in R\right.$ and $\left.\alpha^{2}+k \beta^{2} \in R^{*}\right\}$ is a commutative group, where multiplication is given by

$$
R(\alpha, \beta) \cdot R(\gamma, \delta)=R(\alpha \gamma-k \beta \delta, \alpha \delta+\beta \gamma) .
$$

Let $\mathrm{m}: \mathscr{L}^{\prime}(R) \times \mathscr{L}^{\prime}(R) \rightarrow D_{0}$ denote the mapping

$$
(R[\alpha, \beta, \varepsilon], R[\gamma, \delta, \nu]) \mapsto R(k \alpha \gamma+\beta \delta, \alpha \delta-\beta \gamma) .
$$

Then $\mathrm{m}$ is an angle measure of the affine plane $\Pi^{\prime}(R)$. The mapping

$$
\iota: \mathscr{D} \rightarrow D_{0}, \quad \mathrm{w}(g, h) \mapsto \mathrm{m}(g \xi, h \xi), \quad \text { where } g, h \in \mathscr{L}^{\prime},
$$

is well defined (i.e. does not depend on the choice of $g, h$ ). $\iota$ is a monomorphism of the group $\mathscr{D}$ into the group $D_{0}$. Call $\Omega \in D_{0}$ regular if any two lines $\Sigma, \Gamma$ of $\Pi^{\prime}(R)$ with $\mathrm{m}(\Sigma, \Gamma)=\Omega$ intersect in exactly one point of $\Pi^{\prime}(R)$. Let $D_{0}^{*}$ denote the set of these regular angles $\Omega$. Then $\mathscr{D}^{*} \iota=D_{0}^{*}$. Furthermore, $u \iota=R(0,1)$. 
(iii) Every non-unit of $R$ is a zero-divisor.

(iv) Let $\hat{a} \in \mathscr{P}^{\prime}$ and $g \in \mathscr{L}^{\prime}$ be such that $a-g$. Then $\hat{a} \xi$ distant $g \xi$. ("distant" is defined in Part I.) Let $g, h \in \mathscr{L}^{\prime}$ and $g(u) h$. Then $g \xi$ distant $h \xi$ (definition of (u) see $\S 8$ ). Let $\hat{a}, \hat{b} \in \mathscr{P}^{\prime}$ and $a \mathbf{u} b$. Then $\hat{a} \xi$ distant $\hat{b} \xi$. If $\hat{a}, \hat{b} \in \mathscr{P}^{\prime}, g \mid a, b$ for some $g$ and $\hat{a} \xi$ distant $\hat{b} \xi$ then $a \mathbf{u} b$.

Proof. First, let us recollect

(1) If $y \mid a$ and $\hat{a} \in \mathscr{P}^{\prime}$ then $y \in \mathscr{L}^{\prime}$.

The main result of 9.1 will be obtained from 5.5 of [8]. We apply this theorem to the pseudo-plane. In [8], the technical denotion " $\hat{a}$ fern $g$ " for a point $\hat{a} \in \mathscr{P}^{\prime}$ and a line $g \in \mathscr{L}^{\prime}$ means: Each point $\hat{x} \in \mathscr{P}^{\prime}$ of $g$ has a unique joining line $y$ to $\hat{a}$; and $\mathrm{w}(g, y) \in \mathscr{D}^{*}$; i.e. $g, y$ define a regular angle.

(2) “ $\hat{a}$ fern $g$ ” if and only if $a \rightsquigarrow g$, for any $\hat{a} \in \mathscr{P}^{\prime}$ and $g \in \mathscr{L}^{\prime}$.

Proof. Suppose that " $\hat{a}$ fern $g$ " holds. Let $h$ denote the pseudo-orthogonal of $g$ through $\hat{a}$. Let $\hat{c}$ be the foot. The assumption together with (1) yields $a \mathbf{u} c$ and $\mathrm{w}(g, h) \in \mathscr{D}^{*} .2 .9$ implies $h \mathbf{u} g$. Hence $a \multimap g$. Conversely, let us assume $a-g$. Let $\hat{x} \in \mathscr{P}^{\prime}$ with $x \mid g$. Then $a \mathbf{u} x$. Therefore, $y \mid a, x$ for just one $y \in \mathscr{L}^{\prime}$; cf. (1). Furthermore $y \mathbf{u} g$. 3.4 yields $\mathrm{w}(g, y) \in$ $\mathscr{D}^{*}$.

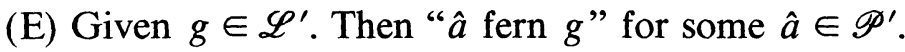

Proof. Let $h \mid u, g$. Then $g h \varpi u$. Select $a$ such that $a \mid h$ and $a \mathbf{u} g h$ and $a \varpi u$; cf. 2.4(ii). Then $\hat{a} \in \mathscr{P}^{\prime}$ and $a \curvearrowleft g$, hence " $a$ fern $g$ "; cf. (2).

Now we have proved that the pseudo-plane fulfills all of the requirements of 5.5 in [8]. This theorem yields the main assertion of 9.1, together with (i) and (ii), after an easy conversion into projective terms. This conversion is carried out in the following small type section.

5.5 of [8] provides an embedding $\zeta$ of $\left(\mathscr{P}^{\prime}, \mathscr{L}^{\prime}, \mid\right)$ into a structure $\mathscr{H}(A, R)$, where $A$ is an algebra over the commutative ring $R$ such that $A=R+R \omega$ for some $\omega \in A$ with $k:=-\omega^{2} \in R^{*}$. The point set of $\mathscr{H}(A, R)$ is $A$. A line is a set $a+R b$, where $a \in A$ and $b \in A^{*}$ (group of units of $A$ ). Incidence is given by inclusion. The angle-measure of a pair of lines is $\mathrm{M}(a+R b, c+R d):=R^{*} b^{-1} d \in A^{*} / R^{*}$. The mapping $\lambda_{M}: A^{*} / R^{*} \rightarrow D_{0}$, $R^{*}(\alpha+\beta \omega) \mapsto R(\alpha, \beta)$ is an isomorphism ( $D_{0}$ is defined in the theorem). $R^{*}(\alpha+\beta \omega)$ $\in A^{*} / R^{*}$ is regular if and only if $\beta \in R^{*}$; cf. [8], 2.1. Let $\lambda$ denote the following 
embedding of $\mathscr{H}(A, R)$ into $\Pi^{\prime}(R)$.

$$
\begin{aligned}
\varepsilon+\eta \omega & \mapsto R(\varepsilon, \eta, 1) \\
(\gamma+\delta \omega)+R(\alpha+\beta \omega) & \mapsto R[-\beta, \alpha,-\alpha \delta+\beta \gamma] .
\end{aligned}
$$

Then $(\mathrm{M}(g, h)) \lambda_{\mathrm{M}}=\mathrm{m}(g \lambda, h \lambda)$ for any two lines $g, h$ of $\mathscr{H}(A, R)$ ( $m$ is defined in 9.1(ii)). Let $\xi:=\zeta \lambda$. Now we obtain 9.1 together with (i) and (ii) from Theorem 5.5 of [8].

Proof of (iii). Suppose $\mu \in R \backslash R^{*}$. We have $g \xi=R[0,1,0]$ for some $g \in \mathscr{L}^{\prime}$ and $\hat{a} \xi=R(\mu, 0,1)$ for some $\hat{a} \in \mathscr{P}^{\prime}$; cf. (i). A line $h \in \mathscr{L}^{\prime}$ through $\hat{a}$ will never satisfy $h \curvearrowleft u$; namely, suppose the contrary. Then (2) implies " $\hat{u}$ fern $h$ ". From 5.5 and 5.2 of [8] we obtain $R(\lambda \mu, 0,1)=$ $R(1,0,1)$ for some $\lambda \in R$; a contradiction. Consequently, $u \mathbf{u} a$ fails to hold. Hence $j \mid a, u$ for some $j \neq g$. Let $j \xi=R[\nu, \chi, 0]$. Then $\nu \neq 0$ and $\mu \nu=0$.

Proof of (iv). Pick $\hat{a} \in \mathscr{P}^{\prime}$ and $g \in \mathscr{L}^{\prime}$ such that $a \curvearrowleft g$. We want to prove $\hat{a} \xi$ distant $g \xi$. Let $h$ be the pseudo-orthogonal of $g$ through $\hat{a}$. Let $\hat{b}$ denote the foot. Then " $\hat{a}$ fern $g$ " by (2). 5.5 of [8] implies that $\hat{a} \xi$ and $\widehat{b \xi}$ have a unique joining line (in the affine plane $\Pi^{\prime}(R)$ ), hence also in $\Pi(R)$ and that $g \xi$ and $h \xi$ intersect uniquely in $\Pi^{\prime}(R)$, hence also in $\Pi(R)$. Due to (iii) this yields $\hat{a} \xi$ distant $\hat{b} \xi$ and $g \xi$ distant $h \xi$; cf. I.2. Thus $\hat{a} \xi$ distant $g \xi$; cf. I.3.

Let $g, h \in \mathscr{L}^{\prime}$ and $g(u) h$. We want to prove $g \xi$ distant $h \xi$. We can assume $a \longmapsto h$ and $a \mid g$ for some $\hat{a} \in \mathscr{P}^{\prime}$. Then $\hat{a} \xi$ distant $h \xi$ by the first statement of (iv). In particular, $g \xi$ distant $h \xi$.

Let $g, h \in \mathscr{L}^{\prime}$ and $a \mid g, h$ for some $\hat{a} \in \mathscr{P}^{\prime}$. If $g \mathbf{u} h$ then $g(u) h$ (cf. 8.1), hence $g \xi$ distant $h \xi$ according to the above proof.

Let $\hat{a}, \hat{b} \in \mathscr{P}^{\prime}$ and $a \mathbf{u} b$. We assert $\hat{a} \xi$ distant $\hat{b} \xi$. Let $g \mid a, b$. Select $h \mid b$ such that $h \mathbf{u} g$. Then $a \curvearrowleft h$ and $h \in \mathscr{L}^{\prime}$, hence $\hat{a} \xi$ distant $h \xi$; cf. first statement of (iv). In particular, $\hat{a} \xi$ distant $\hat{b} \xi$.

Conversely, let us assume $\hat{a}, \hat{b} \in \mathscr{P}^{\prime}, g \mid a, b$ and $\hat{a} \xi$ distant $\hat{b} \xi$. Then $a \mathbf{u} b$, since $h \mid a, b$ implies $h \in \mathscr{L}^{\prime}$ and $g \xi, h \xi \mathrm{I} \hat{a} \xi, \hat{b} \xi$, hence $g \xi=h \xi$ and $g=h$.

REMARK. Let $\Omega=R\left[\alpha_{1}, \alpha_{2}, \alpha_{3}\right] \in \mathscr{L}^{\prime}(R)$ and $\omega=R\left(\omega_{1}, \omega_{2}\right) \in D_{0}$. Then the line $\Gamma:=R\left[\alpha_{1} \omega_{1}-\alpha_{2} \omega_{2}, \alpha_{2} \omega_{1}+k \alpha_{1} \omega_{2}, 0\right]$ satisfies $m(\Omega, \Gamma)=$ $R\left(\omega_{1}, \omega_{2}\right)$.

9.2. Let $g \mid r$ and $j \mid s$ and $r, h, s \mid u$ (hence rhs $\in S$ ). Suppose gu $u$. There is a unique $d \in S$ with $d \mid g r$, rhs. Furthermore,

$$
g h j \in S \Leftrightarrow d \mid j s .
$$




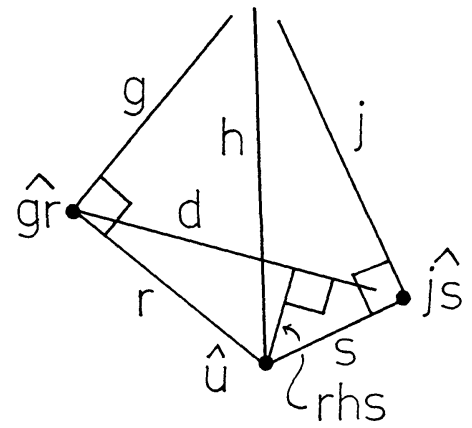

Proof. We have $\alpha:=g h j=(g r)(r h s)(s j)$. If $d \mid g r, r h s, s j$ for some $d$, (E3) yields $\alpha \in S$. Conversely let us assume $\alpha \in S$. guu implies $u \longmapsto g r$ (cf. 2.2), hence $r h s \mathbf{u} g r$. Let $d \mid r h s, g r$. Then $d \mid s j$ by 4.1 .

9.3. (Algebraical description of pseudo-semi-rotations). Let $\hat{y} \in \mathscr{P}^{\prime}$ and $\omega \in \mathscr{D}$. Let $\hat{y} \xi=R\left(y_{1}, y_{2}, 1\right)$ and $\omega \iota=R^{*}\left(\omega_{1}, \omega_{2}\right)$. Let $H=H_{\hat{y}, \omega}$. Then

$$
(g H) \xi=R\left[g_{1}, g_{2}, g_{3}\right]\left(\begin{array}{cll}
\omega_{1} & k \omega_{2} & -k \omega_{2} y_{2} \\
-\omega_{2} & \omega_{1} & \omega_{2} y_{1} \\
0 & 0 & \omega_{1}
\end{array}\right)
$$

for every $g \in \mathscr{L}^{\prime}$ with $g \xi=R\left[g_{1}, g_{2}, g_{3}\right]$. If $\omega \in \mathscr{F}$ then $\omega_{1} \in R^{*}$; hence the linear transformation given by the matrix is bijective.

Proof. Let $j$ denote the pseudo-orthogonal of $g$ through $\hat{y}$. Then $j \xi=R\left[g_{2},-k g_{1}, \gamma\right]$, where $\gamma=k y_{2} g_{1}-y_{1} g_{2}$. The foot $\hat{q}$ is

$$
\hat{q} \xi=R\left(-\gamma g_{2}-k g_{1} g_{3}, \gamma g_{1}-g_{2} g_{3}, k g_{1}^{2}+g_{2}^{2}\right) .
$$

Obviously the line $\Sigma$ obtained by multiplication of $g \xi$ with the above matrix is incident with $\hat{q} \xi$ and satisfies $\mathrm{m}(g \xi, \Sigma)=R\left(\omega_{1}, \omega_{2}\right)$.

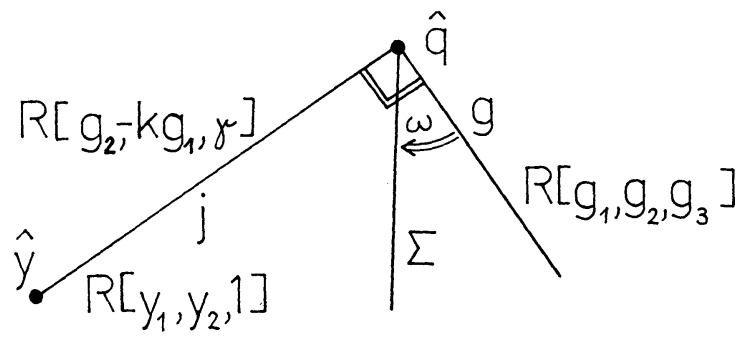


If $\omega \in \mathscr{F}$ then $\omega u \in \mathscr{E}$. Thus $\omega u$ is a regular angle of $\left(\mathscr{P}^{\prime}, \mathscr{L}^{\prime}, 1\right)$. Hence, $(\omega u) \iota=R\left(-k \omega_{2}, \omega_{1}\right)$ is a regular angle of $\Pi^{\prime}(R)$; cf. 9.1 (ii). This means that $\omega_{1} \in R^{*}$.

9.4. Let $g, h, j \in \mathscr{L}^{\prime}$ and $h \mid u$. Let $g \xi=R \Gamma, h \xi=R \Sigma, j \xi=R \Omega$, where $\Gamma, \Sigma, \Omega \in R^{3 *}$. Then

$$
g h j \in S \Leftrightarrow \operatorname{det}(\Gamma, \Sigma, \Omega)=0 .
$$

Proof. Let $r|u, g ; s| u, j$ and $t:=r h s$. Let $d \mid g r, t$; cf. 9.2. Let $\Gamma=$ $\left[g_{1}, g_{2}, g_{3}\right], \Sigma=\left[h_{1}, h_{2}, 0\right], \Omega=\left[j_{1}, j_{2}, j_{3}\right]$. We have

$$
\text { (hs) } \iota=R\left(h_{2} j_{1}-h_{1} j_{2}, h_{1} j_{1}+k^{-1} h_{2} j_{2}\right)
$$

and $d=g H_{\hat{u}, h s} \cdot 9.3$ yields $d \xi=R \Lambda$, where

$$
\begin{aligned}
\Lambda=[- & g_{1} h_{1} j_{2}+g_{1} h_{2} j_{1}-g_{2} h_{1} j_{1}-k^{-1} g_{2} h_{2} j_{2}, \\
& \left.g_{2} h_{2} j_{1}-g_{2} h_{1} j_{2}+g_{1} h_{2} j_{2}+k g_{1} h_{1} j_{1}, g_{3}\left(h_{2} j_{1}-h_{1} j_{2}\right)\right] .
\end{aligned}
$$

Moreover, $(\widehat{j s}) \xi=R \boldsymbol{\theta}$, where $\boldsymbol{\theta}=\left(k j_{1} j_{3}, j_{2} j_{3}, k j_{1}^{2}+j_{2}^{2}\right)$. Hence, $\Lambda \boldsymbol{\theta}=$ $\left(k j_{1}^{2}+j_{2}^{2}\right) \cdot \operatorname{det}(\Gamma, \Sigma, \Omega)$. Since $k j_{1}^{2}+j_{2}^{2}$ is a unit, the following statements are equivalent: $d \mid j s ; \Lambda \theta=0 ; \operatorname{det}(\Gamma, \Sigma, \Omega)=0$. Now, 9.2 yields the assertion.

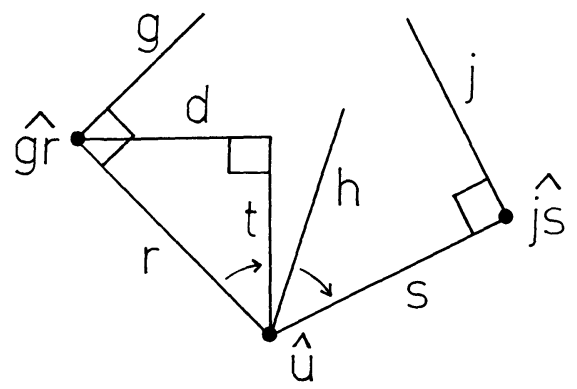

9.5. Let $g, h, j \in \mathscr{L}^{\prime}$ and $g, h \mid c$ and $g \mathbf{u} h$. Let $g \xi=R \Sigma, h \xi=R \Omega$, $j \xi=R \Gamma$, where $\Sigma, \Omega, \Gamma \in R^{3 *}$. Then $j \mid c \Leftrightarrow \operatorname{det}(\Sigma, \Omega, \Gamma)=0$.

Proof. Let $r \mid g, u$ and $s \mid h, u$. Select $y \mid h$ such that $y \mathbf{u} s$ and $y-s$; cf. (E2). Then $\hat{y} \in \mathscr{P}^{\prime}$ as $y \longmapsto u$. From $y \longmapsto s$ and $s \mid u$ follows $y \mathbf{u} u$. Let $d \mid y, u$ and $e:=d u$. $s \longmapsto y$ and $y \mid d$ implies $s d \in \mathscr{E}$, hence $\alpha:=s e \in \mathscr{F}$. Let $H:=H_{\hat{y}, \alpha^{*}} \quad H$ is a bijective pseudo-semi-rotation; cf. 7.3. Moreover, $h H=d \mid u$. From 9.3 we obtain a linear bijective mapping $\psi: R^{3 *} \rightarrow R^{3 *}$ 
such that $(g H) \xi=R(\Sigma \psi),(h H) \xi=R(\Omega \psi),(j H) \xi=R(\Gamma \psi) .9 .3$ yields

(1) $g H \cdot h H \cdot j H \in S \Leftrightarrow \operatorname{det}(\Sigma \psi, \Omega \psi, \Gamma \psi)=0 \Leftrightarrow \operatorname{det}(\Sigma, \Omega, \Gamma)=0$.

We can view $H$ as an automorphism of the incidence structure $(\mathscr{P}, \mathscr{L}, 1)$; cf. 7.5. Since $\hat{c}$ is the only intersection of $g$ and $h, \hat{c} H$ will be the only intersection of $g H$ and $h H$. Therefore, 4.1 implies

$$
j|\hat{c} \Leftrightarrow j H| \hat{c} H \Leftrightarrow g H \cdot h H \cdot j H \in S .
$$

(1) and (2) together yield that $j \mid \hat{c}$ holds if and only if $\operatorname{det}(\Sigma, \Omega, \Gamma)=0$.

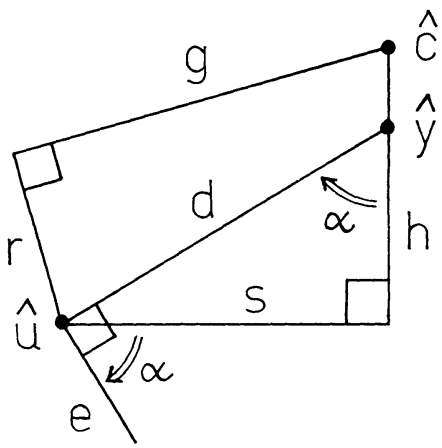

9.6. There is a unique extension of $\xi$ to an embedding of $\left(\mathscr{P}, \mathscr{L}^{\prime}, \mid\right)$ into $\left(\mathscr{P}(R), \mathscr{L}^{\prime}(R), \mathrm{I}\right)$.

Let $\xi$ also denote this extension. If $c \in \mathscr{L}^{\prime}, a, b \mid c$ and $a \mathbf{u} b$ then $\hat{a} \xi$ distant $\hat{b} \xi$.

Proof. Let $c \in \mathscr{P}$. According to 7.4 we may select lines $g, h \in \mathscr{L}^{\prime}$ such that $g, h \mid c$ and $g(u) h$. Let $g \xi=R \Sigma$ and $h \xi=R \Omega$. 9.1 (iv) implies $g \xi$ distant $h \xi$. Thus, $g \xi$ and $h \xi$ intersect in just one point $R(\Sigma \times \Omega)$ of $\Pi(R)$. Let $j \in \mathscr{L}^{\prime}$ and $j \xi=R \Gamma$. By 9.5 the following statements are equivalent.

$$
j \mid c ; \quad \operatorname{det}(\Sigma, \Omega, \Gamma)=0 ; \quad j \xi \operatorname{I} R(\Sigma \times \Omega) .
$$

Hence we can define $\hat{c} \xi:=R(\Sigma \times \Omega)$, and there is no other choice.

Proof of the last statement. Let $v \mid c, u$. Select $w$ such that $w \mid v$ and $w \mapsto c, u$; cf. 2.4. Then $\hat{w} \in \mathscr{P}^{\prime}$ and $w \mathbf{u} a, b$. Let $d \mid a, w$ and $e \mid b, w$. Then $d, e \in \mathscr{L}^{\prime}$ and $\hat{w} \xi$ distant $c \xi$; cf. 9.1(iv). In particular,

$\hat{w} \xi$ distant $\hat{a} \xi$ 
cf. 1.3. We have $d \mathbf{u} e$ (since $d \curvearrowleft b$ ) and $d, e \in \mathscr{L}^{\prime}$ and $w \in \mathscr{P}^{\prime}$; hence $d(4) e$ by 8.1 and

$$
d \xi \text { distant } e \xi
$$

cf. 9.1(iv). (1) and (2) implies $\hat{a} \xi$ distant $e \xi$; cf. I.3. In particular, $\hat{a} \xi$ distant $\hat{b} \xi$.

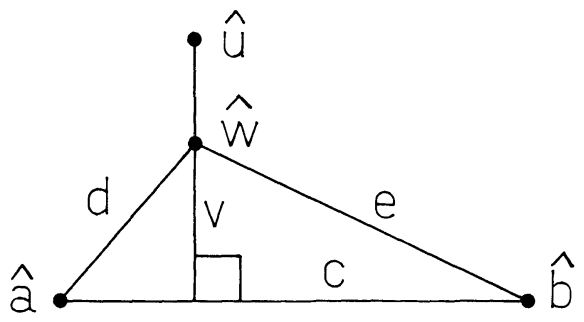

9.7. Finally, we want to attach coordinates to every line of the elliptic plane.

Let $j \in \mathscr{L}$.

Select $r$ such that $r \mid j$ and $r \mathbf{u} u$ (cf. (U)). Let $h \mid u, r$ and $b:=h u$. Select $g \mid u$ such that $g \mathbf{u} h$ and $g \rightsquigarrow h$.

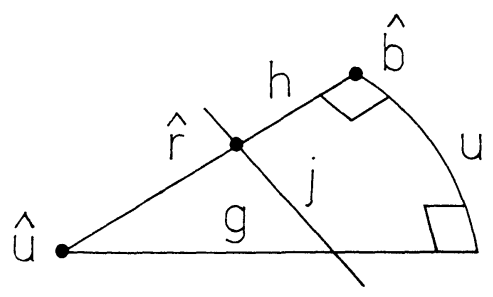

(i) There is a transvection $\tau$ and a point $\hat{a}$ with the following properties. $a \mid h$ and $a \mathbf{u} u ; \hat{u}$ is the center and $g$ is the axis of $\tau ; \hat{a} \tau=\hat{b}$; $j \tau \in \mathscr{L}^{\prime}$.

Proof. Select $t$ such that $t \mid h$ and $t \mathbf{u} u$ and $t-u$; cf. (E2). Then $t \mathbf{u} b$. There is a transvection $\tau$ of $(\mathscr{P}, \mathscr{L}, \mid)$ with center $\hat{u}$ and axis $g$ such that $\hat{r} \tau=\hat{t}$; cf. 8.6. Then $j \tau \in \mathscr{L}^{\prime}$ as $j \tau \mid \hat{t}$ and $\hat{t} \in \mathscr{P}^{\prime}$. Let $\hat{a}:=\hat{b} \tau^{-1}$. Then $a \mid h$ and $a-g$ by 8.7 , hence $a \mathbf{u} u$.

For the rest of this section we select $\tau$ and $a$ according to (i). 
(ii) There is an invertible matrix $A$ such that
$(*) \quad(\hat{y} \tau) \xi=(\hat{y} \xi) A$,
and
$(* *) \quad(y \tau) \xi=A^{-1}(y \xi)$

for every point $\hat{y} \in \mathscr{P}$ and every line $y \in \mathscr{L}^{\prime}$.

Proof. Observe that a point $\hat{y} \in \mathscr{P}$, where $\hat{y} \xi=R\left(y_{1}, y_{2}, y_{3}\right)$, lies on the line $u$ if and only if $y_{3}=0$.

Let $g \xi=R\left[g_{1}, g_{2}, 0\right], \quad \hat{a} \xi=R\left(a_{1}, a_{2}, a_{3}\right), \hat{b} \xi=R\left(a_{1}, a_{2}, 0\right), h \xi=$ $R\left[a_{2},-a_{1}, 0\right]$. Then $h(u) g$ by 8.1 , hence $h \xi$ distant $g \xi$; cf. 9.1 (iv). Thus we can assume $a_{1} g_{1}+a_{2} g_{2}=1$. Let

$$
A:=\left(\begin{array}{ccc}
1 & 0 & -a_{3} g_{1} \\
0 & 1 & -a_{3} g_{2} \\
0 & 0 & 1
\end{array}\right) .
$$

We apply 8.3 in order to prove $(*)$ and $(* *)$. According to 8.3 it is enough to prove $(*)$ and $(* *)$ for $\hat{y} \in \mathscr{N}^{(i)} \cap \mathscr{P}$ and $y \in \mathscr{N}^{(i)} \cap \mathscr{L}^{\prime}$, respectively, (we use the notations introduced in 8.3). We proceed by induction.

For $i=0$ the assertion is easily verified.

Now let us step from $i$ to $i+1$.

Let $\hat{y} \in \mathscr{N}^{(i+1)} \cap \mathscr{P}$. If $\hat{y} \in \mathscr{N}^{(i)}$ then there is nothing to prove. Otherwise, $\hat{y} \mid x_{1}, x_{2}$ and $x_{1}(4) x_{2}$ for some $x_{1}, x_{2} \in \mathscr{N}^{(i)} \cap \mathscr{L}^{\prime}$. Then $x_{1} \xi$ distant $x_{2} \xi$ by $9.1(\mathrm{iv})$, and $\left(x_{j} \tau\right) \xi=A^{-1}\left(x_{j} \xi\right)$ by our assumption. Also $0=(\hat{y} \tau) \xi \cdot\left(x_{j} \tau\right) \xi=(\hat{y} \tau) \xi \cdot A^{-1}\left(x_{j} \xi\right)$ and $0=\hat{y} \xi \cdot x_{j} \xi$. Therefore $(\hat{y} \tau) \xi \cdot A^{-1}=\hat{y} \xi$, i.e. $(\hat{y} \tau) \xi=(\hat{y} \xi) A$.

Let $y \in \mathscr{N}^{(i+1)} \cap \mathscr{L}^{\prime}$. We want to prove $(* *)$ and may assume $y \mid \hat{x}_{1}, \hat{x}_{2}$ for some points $\hat{x}_{1}, \hat{x}_{2} \in \mathscr{N}^{(i)}$ with $x_{1} \mathbf{u} x_{2}$. From 9.6 follows $\hat{x}_{1} \xi$ distant $\hat{x}_{2} \xi$. The assumption yields $\left(\hat{x}_{j} \tau\right) \xi=\left(\hat{x}_{j} \xi\right) A$. Finally, $0=\left(\hat{x}_{j} \tau\right) \xi$ $\cdot(y \tau) \xi=\left(\hat{x}_{j} \xi\right) A \cdot(y \tau) \xi$ and $\hat{x}_{j} \xi \cdot y \xi=0$ for $j=1,2$ implies $A \cdot(y \tau) \xi$ $=y \xi$, hence $(y \tau) \xi=A^{-1}(y \xi)$.

Now let $A$ be a matrix such that (ii) holds. Since $j \tau \in \mathscr{L}^{\prime},(j \tau) \xi \in$ $\mathscr{L}^{\prime}(R)$ is well-defined. $(j \tau) \xi \cdot A^{-1}$ is a line of $\Pi(R)$. We define $j \xi:=(j \tau) \xi \cdot A^{-1}$. Then for any $\hat{y} \in \mathscr{P}$ the following statements are equivalent: $\hat{y} \xi \mathrm{I} j \xi ; \hat{y} \xi \mathrm{I}(j \tau) \xi \cdot A^{-1} ;(\hat{y} \xi) A \mathrm{I}(j \tau) \xi ;($ by $(*))(\hat{y} \tau) \xi \mathrm{I}(j \tau) \xi$; $\hat{y} \tau|j \tau ; \hat{y}| j$. Hence the above definition extends $\xi$ to an embedding of $(\mathscr{P}, \mathscr{L}, \mid)$ into $\Pi(R)$. Clearly, the extension is unique. We summarize our results.

Proposition. There is an embedding $\xi$ of $(\mathscr{P}, \mathscr{L}, \mid)$ into $\Pi(R)$. $\xi$ is an extension of the mapping $\xi$ of 9.1. If $a \mathbf{u} b$ then $\hat{a} \xi$ distant $\hat{b} \xi$ for any $\hat{a}, \hat{b} \in \mathscr{P}$. If $a \varpi j$ then $\hat{a} \xi$ distant $j \xi$ for all $\hat{a} \in \mathscr{P}$ and $j \in \mathscr{L}$. If $g \mathbf{u} h$ then $g \xi$ distant $h \xi$, for all $g, h \in \mathscr{L}$. 
Proof of the last three assertions. Let $a \mathbf{u} b$. Let $j \mid a, b$. If $j \in \mathscr{L}^{\prime}$ then $\hat{a} \xi$ distant $\hat{b} \xi$ by 9.6. Otherwise $j \tau \in \mathscr{L}^{\prime}$ for some transvection $\tau$ according to the previous construction. Then $\hat{a} \tau \xi \operatorname{distant} \hat{b} \tau \xi$, i.e. $\hat{a} \xi \mathrm{A}$ distant $\hat{b} \xi A$, where $A$ is a matrix as in (ii). Thus $\hat{a} \xi \operatorname{distant} \hat{b} \xi$.

Let $a \curvearrowleft j$. We want to prove $\hat{a} \xi$ distant $j \xi$. By the previous consideration we may assume $j \in \mathscr{L}^{\prime}$. Select a point $\hat{b}$ such that $\hat{b} \mid j$ and $b \hookleftarrow u$. Then $a \mathbf{u} b$, hence

$$
\hat{a} \xi \operatorname{distant} \hat{b} \xi
$$

by our first statement. Let $h \mid a, b$. Since $b \in \mathscr{P}^{\prime}$ and $j \mathbf{u} h 8.1$ yields $j(u) h$, hence

$j \xi$ distant $h \xi$

by $9.1($ iv). (1) and (2) implies $\hat{a} \xi$ distant $j \xi$; cf. I.3.

Finally, let us assume $g \mathbf{u} h$. Let $b \mid g, h$ and $a \mid h$ such that $a \mathbf{u} b$. Then $a \curvearrowleft g$ and $\hat{a} \xi$ distant $g \xi$ by what we have proved. In particular, $h \xi$ distant $g \xi ;$ cf. I.3.

10. The bilinear form. We want to describe the polarity $\mathscr{L} \rightarrow \mathscr{P}$, $x \mapsto \hat{x}$ in terms of a bilinear form. Assumptions and notations of previous sections are preserved; in particular, $k \in R^{*}$ is the element introduced in 9.1 .

10.1. Let $x \in \mathscr{L}^{\prime}$ with $x \longmapsto u$ and $x \xi=R\left[x_{1}, x_{2}, x_{3}\right]$. Then $\hat{x} \xi=$ $R\left(k x_{1}, x_{2}, l x_{3}\right)$ for some $l \in R^{*}$.

Proof. Let $y \mid u, x$. Then $y \xi=R\left[x_{2},-k x_{1}, 0\right]$ and $y \xi \mathrm{I} \hat{x} \xi$. Choose $z \mid x$ with $z \longmapsto y$ and $z \mathbf{u} y$. This implies $z \mathbf{u} u$ and $z \longmapsto u$, hence $z \xi=R\left[z_{1}, z_{2}, 1\right]$; cf. 9.1 (iii). Obviously, $y \xi$ distant $z \xi$. Thus the unique point of intersection is

$$
\hat{x} \xi=R\left(k x_{1}, x_{2},-x_{2} z_{2}-k x_{1} z_{1}\right)=R\left(k x_{1}, x_{2}, l x_{3}\right),
$$

where $l:=-x_{3}^{-1}\left(x_{2} z_{2}+k x_{1} z_{1}\right)$. Moreover, $\hat{x} \in \mathscr{P}^{\prime}$, hence $\hat{x} \xi \in \mathscr{P}^{\prime}(R)$. This is equivalent to $l x_{3} \in R^{*}$.

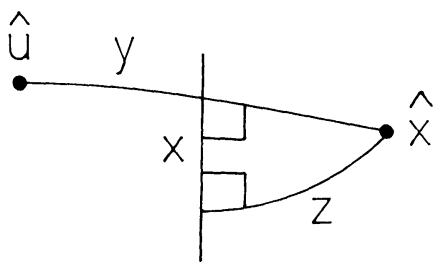


10.2. Let $g \in \mathscr{L}^{\prime}$ with $g_{-u} u g \xi=R\left[g_{1}, g_{2}, g_{3}\right], \hat{g} \xi=R\left(k g_{1}, g_{2}, l g_{3}\right)$. Let $H=H_{\hat{u}, \alpha}$ be a pseudo-semi-rotation with center $\hat{u}, h:=g H, h \xi=$ $R\left[h_{1}, h_{2}, h_{3}\right]$. Then $\hat{h} \xi=R\left(k h_{1}, h_{2}, l h_{3}\right)$.

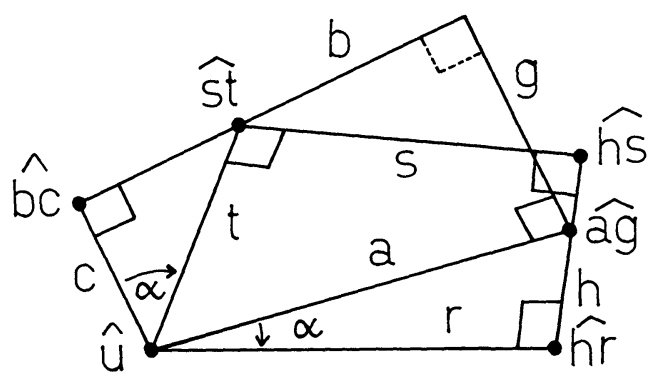

Proof. Let $a \mid g, u$. Then $h \mid \widehat{a g}$ and $a g \longmapsto u$. Let $r \mid h, u$, hence $\alpha=a r$. Choose $s \in S$ with $s \mid h$ and $s \multimap r$ and $s \mathbf{u} r$. This choice implies $s \mathbf{u} u$ and $s \backsim u$. Let $t \mid u, s$ and $c:=\alpha t$. st $\longrightarrow u$ and $c \mid u$ implies $s t \mathbf{u} c$. Let $b \mid c, s t$. From $s, r, a g \mid h$ follows $(s t) c g=s r(a g) \in S$; cf. (E3). Furthermore, st $\mathbf{u} c$ and $b \mid s t, c$. Hence $g \mid b$ by 4.1 . Therefore, $a, b \mid g$, hence $a \xi, b \xi \mathrm{I} \hat{g} \xi$. The rest is a simple calculation involving coordinates. We have

$$
\begin{array}{ll}
g \xi=R\left[g_{1}, g_{2}, g_{3}\right], & a \xi=R\left[-g_{2}, k g_{1}, 0\right] \\
h \xi=R\left[h_{1}, h_{2}, h_{3}\right], & r \xi=R\left[-h_{2}, k h_{1}, 0\right] .
\end{array}
$$

Let

$$
\begin{aligned}
& s \xi=R\left[s_{1}, s_{2}, s_{3}\right], \text { hence } t \xi=R\left[-s_{2}, k s_{1}, 0\right] ; \\
& b \xi=R\left[b_{1}, b_{2}, b_{3}\right], \text { hence } c \xi=R\left[-b_{2}, k b_{1}, 0\right] .
\end{aligned}
$$

Then $\widehat{s t} \xi=R\left(-k s_{1} s_{3},-s_{2} s_{3}, k s_{1}^{2}+s_{2}^{2}\right)$, and $\widehat{s t} \xi \mathrm{I} b \xi$ means that

$$
\left(k s_{1} b_{1}+s_{2} b_{2}\right) s_{3}-\left(k s_{1}^{2}+s_{2}^{2}\right) b_{3}=0 \text {. }
$$

Likewise, $\widehat{a g} \mid h$ implies

$$
\left(k h_{1} g_{1}+h_{2} g_{2}\right) g_{3}-\left(k g_{1}^{2}+g_{2}^{2}\right) h_{3}=0 .
$$

From 9.1(ii) and $c t=\alpha=a r$ we have

$$
\begin{aligned}
& R^{*}\left(k s_{1} b_{1}+s_{2} b_{2},-s_{1} b_{2}+s_{2} b_{1}\right) \\
& \quad=R^{*}\left(k h_{1} g_{1}+h_{2} g_{2},-g_{2} h_{1}+g_{1} h_{2}\right) .
\end{aligned}
$$

In other words

$$
\begin{gathered}
k s_{1} b_{1}+s_{2} b_{2}=\lambda\left(k g_{1} h_{1}+g_{2} h_{2}\right) \text { and } \\
-s_{1} b_{2}+s_{2} b_{1}=\lambda\left(-g_{2} h_{1}+g_{1} h_{2}\right) \quad \text { for some } \lambda \in R^{*} .
\end{gathered}
$$


(3') implies

(4) $\quad\left(k g_{1} b_{1}+g_{2} b_{2}\right)\left(k s_{1}^{2}+s_{2}^{2}\right)=\lambda\left(k h_{1} s_{1}+h_{2} s_{2}\right)\left(k g_{1}^{2}+g_{2}^{2}\right)$.

In the next line, (1), $\left(3^{\prime}\right)$ and (2) yield the first, the second and the third equality:

$$
\begin{aligned}
b_{3} g_{3}\left(k s_{1}^{2}+s_{2}^{2}\right) & =\left(k s_{1} b_{1}+s_{2} b_{2}\right) s_{3} g_{3} \\
& =\lambda\left(k g_{1} h_{1}+g_{2} h_{2}\right) g_{3} s_{3}=\lambda h_{3} s_{3}\left(k g_{1}^{2}+g_{2}^{2}\right) .
\end{aligned}
$$

Finally, (4) and (5) imply

$\lambda\left(k h_{1} s_{1}+h_{2} s_{2}+l h_{3} s_{3}\right)\left(k g_{1}^{2}+g_{2}^{2}\right)=\left(k g_{1} b_{1}+g_{2} b_{2}+l b_{3} g_{3}\right)\left(k s_{1}^{2}+s_{2}^{2}\right)$.

The right side is zero because $b \xi \mathrm{I} \hat{g} \xi=R\left(k g_{1}, g_{2}, l g_{3}\right)$. Therefore, $k h_{1} s_{1}+$ $h_{2} s_{2}+l h_{3} s_{3}=0$. We conclude $\hat{h} \xi=r \xi \times s \xi=R\left(k h_{1}, h_{2}, l h_{3}\right)$.

10.3. Let $j \mid u, g, h$ and $g, h \mathbf{u} u$ and $g \longmapsto u$. Let $g \xi=R\left[g_{1}, g_{2}, g_{3}\right]$ and $h \xi=R\left[h_{1}, h_{2}, h_{3}\right]$. If $\hat{g} \xi=R\left(k g_{1}, g_{2}, \lg _{3}\right)$ then $\hat{h} \xi=R\left(k h_{1}, h_{2}, l h_{3}\right)$.

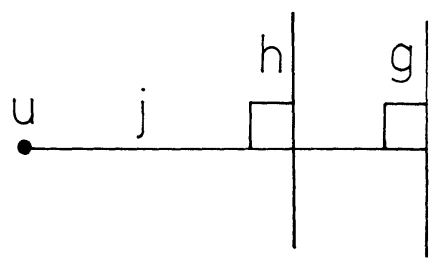

Proof. Select $s \mid h$ with $s \mathbf{u} j$ and $s \longleftarrow j$. Then $s \rightsquigarrow u$ and $s \rightsquigarrow j$, hence also $s \mathbf{u} u$. Let $v \mid u, s$ and $w:=s v$. We have $s \mathbf{u} g j$. Let $m \mid s, g j$ and $n \mid u, m$. There are pseudo-semi-rotations mapping $g$ to $m$ and $w$ to $m$. Thus, by the previous lemma, $\hat{m} \xi=R\left[k m_{1}, m_{2}, l m_{3}\right]$ and $\hat{w} \xi=R\left[k w_{1}, w_{2}, l w_{3}\right](g, m$, $w \smile u$ implies $\left.g_{3}, m_{3}, w_{3} \in R^{*}\right)$. Since $w H=h$ for some pseudo-semi-rotation $H$ whose center is $\hat{u}, 10.2$ finally yields $\hat{h} \xi=R\left(k h_{1}, h_{2}, l h_{3}\right)$.

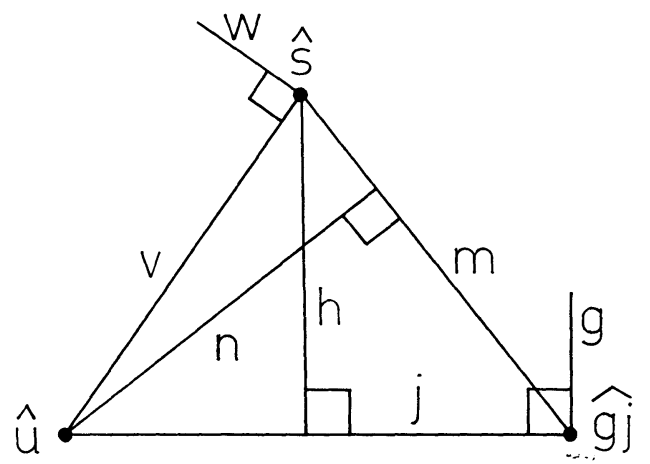


10.4. There is an $l \in R^{*}$ such that $\hat{g} \xi=R\left(k g_{1}, g_{2}, l g_{3}\right)$ for every $g \in \mathscr{L}$ with $g \xi=R\left[g_{1}, g_{2}, g_{3}\right]$.

Proof. Let $x, y \in S$ with $x \xi=R[0,1,0]$ and $y \xi=R[1,0,0]$. Select $r \mid x$ with $r \longmapsto u$ and $r \mathbf{u} u$, and $t \mid y$ with $t \longmapsto u$ and $t \mathbf{u} u$. From 9.1 follows $r \xi=R[1,0, \alpha]$ and $t \xi=R[0,1, \beta]$, where $\alpha, \beta \in R^{*}$. We have $m \mid r x$, ty for some $m$. Moreover, $m \longmapsto u$ and $m u u$. Let $m \xi=R\left(m_{1}, m_{2}, m_{3}\right)$ and $l \in R^{*}$ with $\hat{m} \xi=R\left(k m_{1}, m_{2}, l m_{3}\right)$; cf. 10.1. From 10.2 we conclude $\hat{r} \xi=$ $R(k, 0, l \alpha)$ and $\hat{t} \xi=R(0,1, l \beta)$.

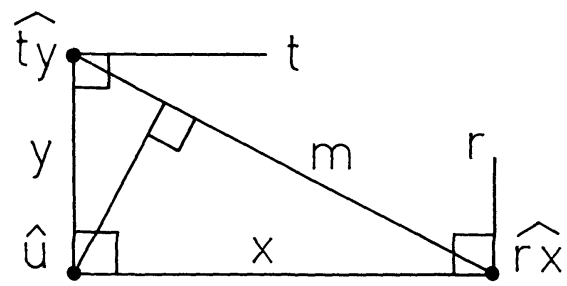

We claim $\hat{g} \xi=R\left(k g_{1}, g_{2}, l_{3}\right)$ for every $g \in \mathscr{L}$, where $g \xi=$ $R\left[g_{1}, g_{2}, g_{3}\right]$.

First, let us assume $g \in \mathscr{L}^{\prime}$, i.e. $g \mathbf{u} u$. Let $h \mid u, g$. Then $h \xi=$ $R\left[-g_{2}, k g_{1}, 0\right]$. Let $v \mid r x, h$ and $w \mid t y, h$. Then $v \xi=R\left[g_{1}, g_{2}, \alpha g_{1}\right]$ and $w \xi=R\left[g_{1}, g_{2}, \beta g_{2}\right]$. By $10.1, \hat{g} \xi=R\left(k g_{1}, g_{2}, l^{\prime} g_{3}\right)$ for some $l^{\prime} \in R$. Thus $\hat{v} \xi=R\left(k g_{1}, g_{2}, l^{\prime} \alpha g_{1}\right)$ and $\hat{w} \xi=R\left(k g_{1}, g_{2}, l^{\prime} \beta g_{2}\right)$; cf. 10.3.

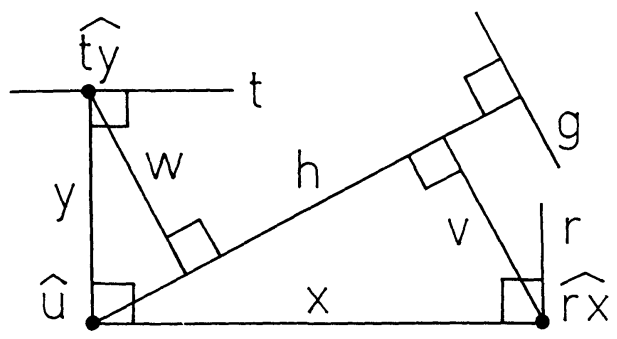

On the other hand, $v$ is the image of $r$ under a pseudo-semi-rotation with center $\hat{u}$. Hence $\hat{v} \xi=R\left(k g_{1}, g_{2}, l \alpha g_{1}\right)$; cf. 10.2. The same conclusion, with $t$ instead of $r$, yields $\hat{w} \xi=R\left(k g_{1}, g_{2}, l \beta g_{2}\right)$. Using $k g_{1}^{2}+g_{2}^{2} \in$ $R^{*}$ and $\alpha \in R^{*}$ we obtain $l g_{1}=l^{\prime} g_{1}$. Likewise, $l g_{2}=l^{\prime} g_{2}$. Hence $l=l^{\prime}$.

Finally, let $g$ be an arbitrary line $\in \mathscr{L}$. From 7.4 we get $v, w \in \mathscr{L}^{\prime}$ and $c \in \mathscr{P}^{\prime}$ with $v, w|g ; v \mathbf{u} w ; c| w ; c \curvearrowleft v$. From 9.1(iv) we obtain $\hat{c} \xi$ distant $v \xi$. Hence $v \xi$ distant $w \xi$. Thus, in $\Pi(R)$ the lines $v \xi, w \xi$ have just 
one point of intersection, namely $\hat{g} \xi$. Let $v \xi=R\left[v_{1}, v_{2}, v_{3}\right]$ and $w \xi=$ $R\left[w_{1}, w_{2}, w_{3}\right]$. Then $\hat{v} \xi=R\left(k v_{1}, v_{2}, l v_{3}\right)$ and $\hat{w} \xi=R\left(k w_{1}, w_{2}, l w_{3}\right)$ by the previous special case. From $v \xi$ distant $w \xi$ and $k, l \in R^{*}$ follows $\hat{v} \xi$ distant $\hat{w} \xi$. Hence

$$
\begin{aligned}
g \xi & =\hat{v} \xi \times \hat{w} \xi \\
& =R\left[l\left(v_{2} w_{3}-v_{3} w_{2}\right), k l\left(v_{3} w_{1}-v_{1} w_{3}\right), k\left(v_{1} w_{2}-v_{2} w_{1}\right)\right] .
\end{aligned}
$$

Finally,

$$
\begin{aligned}
\hat{g} \xi & =v \xi \times w \xi \\
& =R\left[k l\left(v_{2} w_{3}-v_{3} w_{2}\right), k l\left(v_{3} w_{1}-v_{1} w_{3}\right), k l\left(v_{1} w_{2}-v_{2} w_{1}\right)\right]
\end{aligned}
$$

10.5. We define a symmetric bilinear form $f: R^{3} \times R^{3} \rightarrow R, \quad\left(\left(a_{1}, a_{2}, a_{3}\right),\left(b_{1}, b_{2}, b_{3}\right)\right) \mapsto l a_{1} b_{1}+k l a_{2} b_{2}+k a_{3} b_{3}$, where $k$ and $l$ stem from 10.4 .

Proposition. Let $a, b \in S$ and $\hat{a} \xi=R A, \hat{b} \xi=R B$.

(a) $a \mid b$ if and only if $f(A, B)=0$.

(b) $f(A, A) \in R^{*}$.

Proof. (a) Let $A=\left(a_{1}, a_{2}, a_{3}\right)$ and $B=\left(b_{1}, b_{2}, b_{3}\right)$. Then $b \xi=$ $R\left[l b_{1}, k l b_{2}, k b_{3}\right]$; cf. 10.4. The following statements are equivalent. $a \mid b$; $\hat{a} \mid b ; \hat{a} \xi \mathrm{I} b \xi ; f(A, B)=0$. (b) We have $a \multimap a$ and thus $\hat{a} \xi$ distant $a \xi$; cf. the proposition in $\$ 9.7$. This means that $f(A, A) \in R^{*}$; cf. part I.

10.6. $f(A, A) \in R^{*}$ for any point $R A$ of $\Pi(R)$; i.e. $\Pi(R, f)$ is an elliptic plane in the sense of $\S 1$.

Proof. Let $R A$ be a point of $\Pi(A)$ and let $\mathrm{m}$ be an arbitrary maximal ideal of $R$. We want to prove

$$
f(A, A) \notin \mathfrak{m} .
$$

Let - denote the canonical homomorphisms

$$
\begin{aligned}
& -: R \rightarrow \bar{R}:=R / \mathrm{m} \\
& -: R^{3} \rightarrow \bar{R}^{3}, \quad R^{3 *} \rightarrow \bar{R}^{3 *} .
\end{aligned}
$$

Let $\alpha$ denote the canonical homomorphism of $\Pi(R)$ onto the projective plane $\Pi(\bar{R})$ over the field $\bar{R}$ :

$$
\begin{aligned}
& \alpha: R X \mapsto \bar{R} \bar{X} \\
& \alpha: R Y \mapsto \bar{R} \bar{Y},
\end{aligned}
$$


where $R X$ is an arbitrary point and $R Y$ a line of $\Pi(R)$. First we will show

$(* *)$

$$
\mathscr{P} \xi \alpha=\mathscr{P}(R) \alpha=\mathscr{P}(\bar{R})
$$

Proof of $(* *)$. Let $\bar{R} \bar{B} \in \mathscr{P}(\bar{R})$, where $B \in R^{3}$. We claim $\bar{R} \bar{B} \in \mathscr{P} \xi \alpha$. One can assume $B=\left(b_{1}, b_{2}, 1\right)$ or $B=(1,0,0)$ or $B=(\lambda, 1,0)$ for some $b_{1}, b_{2} \in R$ or $\lambda \in R$, respectively. In the first case $R B \in \mathscr{P}^{\prime}(R)=\mathscr{P}^{\prime} \xi \subseteq$ $\mathscr{P} \xi$, hence $\bar{R} \bar{B}=(R B) \alpha \in \mathscr{P} \xi \alpha$; cf. 9.1(i). Let $g, h \in \mathscr{L}$ be lines such that $g \xi=R[0,1,0]$ and $h \xi=R[1,0,0]$; cf. 9.1(i). In the second case follows $R B=R(1,0,0)=(\widehat{g u}) \xi \in \mathscr{P} \xi$, hence $(R B) \alpha \in \mathscr{P} \xi \alpha$. Now we consider the last case $B=(\lambda, 1,0)$. The line $J:=R[1,-\lambda, 0] \in \mathscr{L}(R)$ joins the point $\hat{u} \xi$ to the point $R B$. Furthermore, $m(g \xi, J)=R(\lambda, 1)$ is a regular angle, since $(1,0),(\lambda, 1)$ constitute a $R$-basis of $R^{2}$. Thus $\mathrm{m}(h \xi, J)$ $=\mathrm{w}(h, j) \iota=\mathrm{m}(h \xi, j \xi)$ for some $j \in \mathscr{L}^{\prime}$ with $j \mid u$; cf. 9.1(ii), $\mathscr{D}^{*} \iota=D_{0}^{*}$. This implies $j \xi=J$. Finally, $R B=(\widehat{j u}) \xi \in \mathscr{P} \xi$ since $(\widehat{j u}) \xi I J, u \xi$. Thus we have proved $\bar{R} \bar{B}=(R B) \alpha \in \mathscr{P} \xi \alpha$ in each of the three cases.

Now we deduce (*). From (**) follows that $(R A) \alpha=\bar{R} \bar{A}=(\hat{a} \xi) \alpha$ for some $\hat{a} \in \mathscr{P}$. Let $\hat{a} \xi=R B$, where $B \in R^{3}$. Then $\bar{R} \bar{A}=\bar{R} \bar{B}$ and $f(B, B)$ $\in R^{*}$; cf. 10.5. Thus, $\bar{A}=\bar{\mu} \bar{B}$ for some $\mu \in R \backslash \mathfrak{m}$, and $\overline{f(A, A)}=$ $\bar{\mu}^{2} \cdot \overline{f(B, B)} \neq \overline{0}$. This concludes the proof.

11. The group of motions. In this section we assume that $(G, S)$ satisfies all of our five axioms. For the first time we shall also use (M).

\subsection{If $g \mathbf{u} h$ and $g \longrightarrow h$ then $g \mathbf{u} g^{h}$.}

Proof. Let $a \mid g, h$. Suppose that $g \mathbf{u} g^{h}$ does not hold. Then $b \mid g, g^{h}$ and $b \backsim a$ for some $b \neq a$; cf. 2.6. In particular, $b u h$. Let $j \mid b, h$. Then $b \backsim h j$, and $g \mathbf{u} j$ since $g \curvearrowleft h$. Together with $b, b^{h} \mid g, j$ follows $b=b^{h}=b^{h j}$. (M) yields $b=h j$. Thus, $a, b \mid g, h$. Finally, $g \mathbf{u} h$ implies the contradiction $a=b$.

\section{2. $2 \in R^{*}$.}

Proof. Let $g \in \mathscr{L}$ such that $g \xi=R[0,1,0] \mathrm{I} \hat{u} \xi=R(0,0,1)$. Select $j$ such that $j \mid \hat{u}$ and $j \mathbf{u} g$ and $j-g$. Then $j \mathbf{u} g, h$, where $h:=u g$. Thus, $j \xi$ distant $g \xi, h \xi$; cf. the proposition in $\$ 9.7$. Therefore $j \xi=R[1, \lambda, 0]$ for some $\lambda \in R^{*}$. Let $q:=j^{g}$. Then

$$
R(\lambda, 1)=\mathrm{m}(j \xi, g \xi)=(\mathrm{w}(j, g)) \iota=(\mathrm{w}(g, q)) \iota=\mathrm{m}(g \xi, q \xi) ;
$$

cf. 9.1(ii). Thus, $q \xi=R[1,-\lambda, 0]$. 11.1 implies $j \mathbf{u} q$, hence $j \xi$ distant $q \xi$; cf. 9.7. This means that $\lambda+\lambda \in R^{*}$. 
We have proved that $\Pi(R, f)$ is an elliptic plane over the ring $R$ in the sense of Part I and that 2 is a unit (10.6 and 11.2). In particular, the reflection $\sigma_{\Omega}$ in any point $\Omega$ of $\Pi(R, f)$ is well-defined.

Every $\alpha \in G$ induces an automorphism $\tilde{\alpha}$ of the elliptic plane $(\mathscr{P}, \mathscr{L}, \mathrm{I})$ :

$$
\begin{aligned}
\tilde{\alpha}: \quad \hat{x} & \mapsto \widehat{x^{\alpha}} \text { for } \hat{x} \in \mathscr{P}, \quad \text { and } \\
y & \mapsto y^{\alpha} \text { for } y \in \mathscr{L} .
\end{aligned}
$$

$\tilde{\alpha}$ is called the motion induced by $\alpha$. The mapping ${ }^{\sim}$ is a homomorphism of the group $G$ into the group of automorphisms of $(\mathscr{P}, \mathscr{L}, \mid)$, and the kernel is the center $C_{G}$ of $G$. In 5.5 we have proved $C_{G}=1$. This yields

11.3. The mapping $\tilde{}{ }^{\sim} G \rightarrow \tilde{G}$ is an isomorphism of the group $G$ onto the subgroup $\tilde{G}$ of the group of automorphisms of $(\mathscr{P}, \mathscr{L}, \mid)$.

Let $(\mathscr{G}, \mathscr{S})$ denote the group of motions of the elliptic plane $\Pi(R, f)$; cf. Part I. Hence $\mathscr{S}=\left\{\sigma_{\Omega}: \Omega \in \mathscr{P}(R)\right\}$, and $\mathscr{G}$ is the group generated by $\mathscr{S}$.

If $\Omega$ is a point of $\Pi(R, f)$ and $\Gamma$ is its polar line (cf. Part I) then we define $\sigma_{\Gamma}:=\sigma_{\Omega}$. In particular, $\sigma_{a \xi}=\sigma_{\hat{a} \xi}$ for any $a \in \mathscr{L}$.

11.4. Let $g \in S$ and $\hat{x} \in \mathscr{P}$. Then $\left(\widehat{x^{g}}\right) \xi=(\hat{x} \xi) \sigma_{g \xi}$. In other words, $\tilde{g} \xi=\xi \sigma_{g \xi}$ for every $g$.

The proof consists of four steps.

11.4(a). Let $c \mathbf{u} g$ and $c \backsim g$. Then $\left(c^{g}\right) \xi=(c \xi) \sigma_{g \xi}$.

Proof. Let $r \mid c$, g. Select $r^{\prime}$ such that $r^{\prime} \mid g ; r^{\prime} \mathbf{u} r$ and $r^{\prime} \longmapsto r$; cf. (E2). Then $c \mathbf{u} r^{\prime}$. Let $c^{\prime} \mid c, r^{\prime}$ and $a:=c c^{\prime}$. Then $a \mathbf{u} g$. Let $h \mid a, g . r^{\prime} \longmapsto r$ implies $c^{\prime} \mathbf{u} r$. Together with $c \mathbf{u} g$ follows $c^{\prime} \curvearrowleft g$. Furthermore, $c^{\prime} \mathbf{u} g$ since $c \curvearrowleft g$.

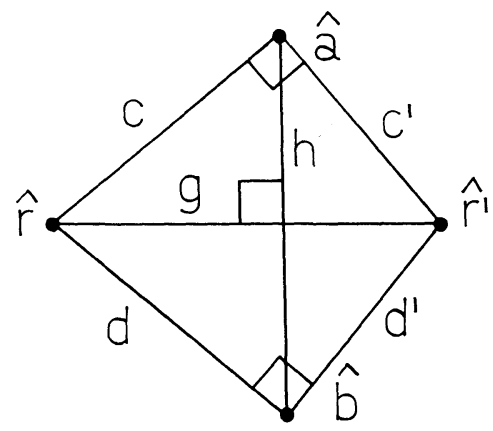


Hence $d^{\prime}:=c^{\prime g} \mathbf{u} c^{\prime}$; cf. 11.1. The proposition of $\$ 9.7$ yields $d^{\prime} \xi$ distant $c^{\prime} \xi$. This implies

$$
\sigma_{d^{\prime} \xi} \mathbf{u} \sigma_{c^{\prime} \xi} \text { in }(\mathscr{G}, \mathscr{S}) \text {. }
$$

Let $b:=a^{g}$ and $d:=c^{g}$. Then $b=d d^{\prime} \mid h$. Hence $\hat{a} \xi, \hat{g} \xi, \hat{b} \xi \mathrm{I} h \xi$ and

$$
\sigma_{a \xi} \sigma_{g \xi} \sigma_{b \xi} \in \mathscr{S}
$$

by I.15 and I.10. The analogue conclusion applies to the lines $c \xi, g \xi, d \xi$ since they pass through the point $\hat{r} \xi$. Thus, $\sigma_{c \xi} \sigma_{g \xi} \sigma_{d \xi}=\sigma_{\Gamma}$ for some line $\Gamma$ of $\Pi(R, f)$ through $\hat{r} \xi$. The identity

$$
\sigma_{c^{\prime} \xi} \sigma_{\Gamma} \sigma_{d^{\prime} \xi}=\sigma_{c^{\prime} \xi} \sigma_{c \xi} \sigma_{c \xi} \sigma_{\Gamma} \sigma_{d \xi} \sigma_{d \xi} \sigma_{d^{\prime} \xi}=\sigma_{a \xi} \sigma_{g \xi} \sigma_{b \xi}
$$

together with (2) yields

$$
\sigma_{c^{\prime} \xi} \sigma_{\Gamma} \sigma_{d^{\prime} \xi} \in \mathscr{S} \text {. }
$$

$r \curvearrowleft r^{\prime}$ implies $\hat{r} \xi$ distant $r^{\prime} \xi$; cf. 9.7. In particular, $\Gamma$ distant $r^{\prime} \xi$; cf. I.3. Thus

$$
\sigma_{\Gamma} \mathbf{u} \sigma_{r^{\prime} \xi} \text { in }(\mathscr{G}, \mathscr{S}) .
$$

Now we use (1), (4) and (5) in order to apply 2.10 to $(\mathscr{G}, \mathscr{S})$. (Note that 2.10 does not require $(\mathrm{U})) .2 .10$ yields $\sigma_{\Gamma} \mid \sigma_{r^{\prime} \xi}$, hence $\Gamma \mathrm{I} \hat{r}^{\prime} \xi$; cf. the proposition in I.15. Thus we obtain

$$
\Gamma, g \xi \mathrm{I} \hat{r} \xi, \widehat{r^{\prime}} \xi \text { and } \hat{r} \xi \operatorname{distant} \widehat{r^{\prime}} \xi \text {. }
$$

This implies $\Gamma=g \xi$, hence $\sigma_{d \xi}=\sigma_{c \xi}^{\sigma \xi}$. I.8(iv) yields $d \xi=(c \xi) \sigma_{g \xi}$. This is the assertion.

11.4(b). $\left(\widehat{x^{g}}\right) \xi=(\hat{x} \xi) \sigma_{g \xi}$ for each pair $x, g$ with $x \mathbf{u} g$.

Proof. Let $h \mid g, x$. Select $c$ such that $c \mid x ; c \mathbf{u} h$ and $c \longmapsto h$. Then $d:=c x$ satisfies $d \mid x ; d \mathbf{u} h ; d \rightsquigarrow h$. Also $c \mathbf{u} g$ and $d \mathbf{u} g$. From $c|x ; x| h ; h \mid g ; c \mathbf{u} h$ and $x \mathbf{u g}$ follows $c \longmapsto g$. Likewise, $d \longmapsto g$. 11.4(a) shows $\left(c^{g}\right) \xi=(c \xi) \sigma_{g \xi}$ and $\left(d^{g}\right) \xi=(d \xi) \sigma_{g \xi}$. Hence $\widehat{\left(x^{g}\right)} \xi,(\hat{x} \xi) \sigma_{g \xi} \mathrm{I}\left(c^{g}\right) \xi,\left(d^{g}\right) \xi$. Furthermore, $\left(c^{g}\right) \xi$ distant $\left(d^{g}\right) \xi$. Thus, $\left(\widehat{x^{g}}\right) \xi=(\hat{x} \xi) \sigma_{g}$.

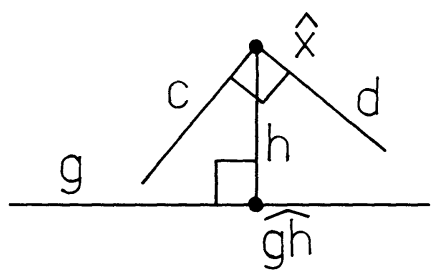




\section{4(c). $\left(y^{g}\right) \xi=(y \xi) \sigma_{g \xi}$ for each $y, g$ with $y \mathbf{u} g$.}

Proof. Let $x \mid g, y$. Select $x^{\prime}$ such that $x^{\prime} \mid y$ and $x^{\prime} \mathbf{u} x$ and $x^{\prime} \curvearrowleft x$. Then $x, x^{\prime} \mathbf{u} g . x, g$ and $x^{\prime}, g$ fulfill the requirements of $11.4(\mathrm{~b})$. Furthermore, $\hat{x} \xi$ distant $\widehat{x^{\prime}} \xi$. This yields the assertion.

Now we are ready to prove 11.4 . Given $g, x$. Select $y$ such that $y \mid x$ and $y \mathbf{u} g$; cf. (U). Let $a \mid g, y$. Select $z$ such that $z \mid a$ and $z \smile g, y$; cf. 2.4(i). Then $x \mathbf{u} z$. Let $y^{\prime} \mid x, z$. The two pairs $y, g$ and $y^{\prime}, g$ satisfy the assumptions of $11.4(\mathrm{c})$. Furthermore, $y \mathbf{u} y^{\prime}$ since $y \curvearrowleft z$. Hence $y \xi$ distant $y^{\prime} \xi$, and $11.4(\mathrm{c})$ implies $\left(\widehat{x^{g}}\right) \xi=(\hat{x} \xi) \sigma_{g \xi}$.

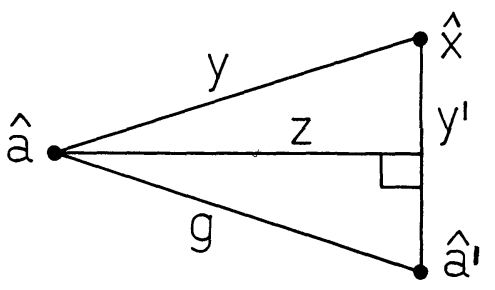

11.5. There is a monomorphism $\sigma: G \rightarrow \mathscr{G}$ such that $\sigma: g \mapsto \sigma_{g \xi}$ for every $g$,

and $\mathscr{F}(R, f) \subseteq G \sigma$.

Proof. We want to extend the mapping $\sigma: S \rightarrow \mathscr{S}, g \mapsto \sigma_{g \xi}$, to a homomorphism of $G$ into $\mathscr{G}$. Let $a_{1}, \ldots, a_{n} \in S$. Let $\alpha:=a_{1} \cdots a_{n}$ and $\gamma:=\sigma_{a_{1} \xi} \cdots \sigma_{a_{n} \xi}$. Applying $11.4 n$ times we obtain

(*) $\quad \tilde{\alpha} \xi=\tilde{a}_{1} \cdots \tilde{a}_{n} \xi=\xi \gamma ; \quad$ i.e. $\overline{x^{a_{1} \cdots a_{n}} \xi}=(\hat{x} \xi) \gamma \quad$ for every $x$.

Suppose $\alpha=1$. We want to prove $\gamma=$ id. From (*) follows that $\Omega=\Omega \gamma$ for every $\Omega \in \mathscr{P} \xi . \gamma$ is a linear mapping $R^{3} \rightarrow R^{3}$, and the points $R(0,0,1), R(1,0,0), R(0,1,0), R(1,1,1)$ are elements of $\mathscr{P} \xi$; cf. 9.1(i) and 9.7. Hence they are fixed points of $\gamma$. It follows that there is some $\lambda \in R^{*}$ such that $X \gamma=\lambda X$ for every $X \in R^{3}$. We obtain $\lambda^{2}=1$ since $\gamma$ is an orthogonal mapping, and $\lambda^{3}=1$ since $\operatorname{det} \gamma=1$. Thus $\lambda=1$ and $\gamma=\mathrm{id}_{R^{3}}$. Now we have proved that $\sigma$ can be extended to a homomorphism $\sigma$ of $G$ into $\mathscr{G}$, namely

$$
\sigma: \alpha=a_{1} \cdots a_{n} \mapsto \gamma=\sigma_{a_{1} \xi} \cdots \sigma_{a_{n} \xi}
$$

If $\alpha \in$ kernel $\sigma$ then $\gamma=\mathrm{id}$, and (*) implies $\hat{x} \tilde{\alpha}=\hat{x}$ for every $\hat{x} \in \mathscr{P}$. Since the mapping ${ }^{\sim}$ is injective (cf. 11.3) we conclude $\alpha=1$. Thus we have proved that $\sigma$ is injective. 
Finally, let $\mu \in \mathscr{F}(R, f)$. We have $\hat{u} \xi=R(0,0,1)$ and $g \xi=R[0,1,0]$ for some $g$; cf. 9.1(i). From 1.17(a) one obtains $\mu=\sigma_{\Omega} \sigma_{g \xi} \sigma_{\Gamma}$, where $\Gamma$ is a line through $\hat{u} \xi$ with $g \xi$ distant $\Gamma$, and $\Omega$ is a point with $\Omega$ distant $u \xi$. The angle $\mathrm{m}(g \xi, \Gamma)$ is regular (cf. 9.1(ii)) since the lines $\Gamma$ and $g \xi$ intersect in just one point of the affine plane $\Pi^{\prime}(R)$ and since every non-unit of $R$ is a zero-divisor; cf. 9.1(iii) and [3], 2.3. 9.1(ii) yields $\Gamma=j \xi$ for some $j \in \mathscr{L}$. Also $\Omega=\hat{q} \xi$ for some $q$, since each affine point of $\Pi(R)$ is the $\xi$-image of some point of $(\mathscr{P}, \mathscr{L}, \mathrm{I})$; cf. 9.1(i). We conclude $\mu=(q g j) \sigma \in G \sigma$.

Appendix: Fixed point theorems. Let $(G, S)$ satisfy the system of axioms introduced in $\$ 1$ of Part II. We reformulate 4.1:

1. Let $a b=c d$ and $c, d \mid q$. If $a \mathbf{u} b$ then $c \mathbf{u} d$ and $q \mid a, b$

2. Let $a, b \mid d$ and $a \mathbf{u} b$ and $c \mathbf{u} d$. Then $c^{a b}=c$ implies $a b=d \mid c$.

Proof. Let $g \mid c, d$. Then $h:=g a b \in S$ since $g, a, b \mid d$. Also $h \mid d$. 1 . implies $h \mathbf{u} g$. Furthermore, $c \mathbf{u} d$. Hence $c-h$, and $c=c^{a b}=c^{g h}=c^{h}$ yields $c=h \mid d$; cf. (M). Therefore, $g \mid h=c$ and $g, h \mid d$. Hence $d=g h=$ $a b$; cf. 2.2(b).

3. Let $a, b \mid d$ and $a \mathbf{u} b$ and $c \rightsquigarrow d$. If $c^{a b}=c$ then $c=d$.

Proof. (E1) implies $c u a, b$. Let $g \mid a, c$ and $h \mid b, c$. We have $c \mid g, h$ and $c^{a}=c^{b} \mid g, h$. Furthermore, $g \mathbf{u} h$ by 2.9. Thus $c=c^{a}=c^{g a}$, and $c \longmapsto g a$ by 2.2. Therefore $c=g a$ by (M). Likewise, $c=h b$. Thus $c \mid a, b$, and $a \mathbf{u} b$ yields $c=d$.

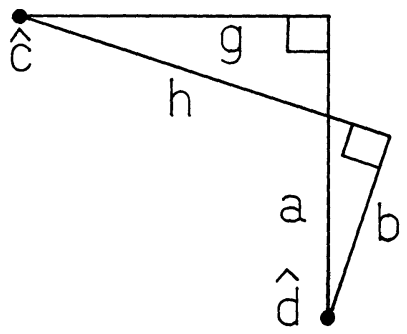

4. Let $a \rightsquigarrow b$ and $c \mathbf{u} a, b$. If $c^{a b}=c$ then $a b=g h$, where $g \mid a, c$ and $h \mid b, c$.

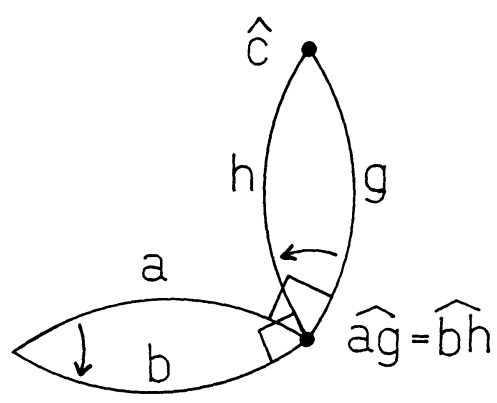


Proof. $a \longmapsto b$ implies $g a \mathbf{u} b$. Let $d \mid g a, b$. Then $c \mathbf{u} d$, since $c \mathbf{u} a$ implies $c \longmapsto g a$. Furthermore, $c=c^{a b}=c^{g a b}$. We apply 2 (with $g a, b, c, d$ instead of $a, b, c, d)$ and obtain $g a b=d \mid c$. Thus, $d \mid c, b$ and $h \mid c, b$ and $c \mathbf{u} b$. Hence $d=h$, and finally $a b=g h$.

\section{REFERENCES}

[1] F. Bachmann, Aufbau der Geometrie aus dem Spiegelungsbegriff, 2. Auflage, Springer 1973.

[2] W. Benz, Ebene Geometrie über einem Ring, Math. Nachr., 59 (1974), 163-193.

[3] F. Knüppel, Äquiforme Ebenen über kommutativen Ringen und singuläre Prä-Hjelmslevgruppen, Abh. Math. Sem. Univ. Hamburg, 53 (1983), 229-257.

[4] R. Lingenberg, Euklidische Pseudoebene über einer metrischen Ebene, Abh. Math. Sem. Univ. Hamburg, 22 (1958), 114-130.

[5] A. Micali and $\mathrm{Ph}$. Revoy, Modules quadratiques, Bulletin de la Soc. Math. de France, No. 63 (1979).

[6] J. Milnor and D. Husemoller, Symmetric Bilinear Forms, Springer 1973.

[7] E. Salow, Einbettung von Hjelmslev-Gruppen in orthogonale Gruppen über kommutativen Ringen, Math. Z., 134 (1973), 143-170.

[8] _ Verallgemeinerte Halbdrehungsebenen, Geom. Ded., 13 (1982), 67-85.

[9] E. M. Schröder, Modelle ebener metrischer Ringgeometrien, Abh. Math. Sem. Univ. Hamburg, 48 (1979), 139-170.

[10] F. Veldkamp, Projective planes over rings of stable rank 2, Geom. Ded., 11 (1981), 285-308.

Received November 14, 1984 and in revised form June 25, 1985. The first-named author is indebted to the University of Toronto for support during a stay in 1983.

UNIVERSITY OF KIEL

OLSHAUSENSTRASSE 40-60

2300 Kiel 1, W. GERMANY 


\title{
PACIFIC JOURNAL OF MATHEMATICS EDITORS
}

\author{
V. S. VARADARAJAN \\ (Managing Editor) \\ University of California \\ Los Angeles, CA 90024 \\ HERBERT ClEMENS \\ University of Utah \\ Salt Lake City, UT 84112 \\ R. FINN \\ Stanford University \\ Stanford, CA 94305
}

\author{
HERMANN FLASCHKA \\ University of Arizona \\ Tucson, AZ 85721 \\ RAMESH A. GANGOLLI \\ University of Washington \\ Seattle, WA 98195 \\ VAUGHAN F. R. JONES \\ University of California \\ Berkeley, CA 94720 \\ ROBION KIRBY \\ University of California \\ Berkeley, CA 94720
}

C. C. MOORE

University of California

Berkeley, CA 94720

H. SAMELSON

Stanford University

Stanford, CA 94305

HAROLD STARK

University of California, San Diego

La Jolla, CA 92093

\section{ASSOCIATE EDITORS}

\author{
R. AREnS \\ E. F. BECKENBACH \\ B. H. NEUMANN \\ F. WOLF \\ K. YOSHIDA \\ (1906-1982)

\section{SUPPORTING INSTITUTIONS}

\begin{abstract}
UNIVERSITY OF ARIZONA
UNIVERSITY OF BRITISH COLUMBIA

UNIVERSITY OF CALIFORNIA

MONTANA STATE UNIVERSITY

UNIVERSITY OF NEVADA, RENO

NEW MEXICO STATE UNIVERSITY

OREGON STATE UNIVERSITY
\end{abstract} \\ CALIFORNIA INSTITUTE OF TECHNOLOGY \\ UNIVERSITY OF OREGON \\ UNIVERSITY OF SOUTHERN CALIFORNIA \\ STANFORD UNIVERSITY \\ UNIVERSITY OF HAWAII \\ UNIVERSITY OF TOKYO \\ UNIVERSITY OF UTAH \\ WASHINGTON STATE UNIVERSITY \\ UNIVERSITY OF WASHINGTON
}

The Supporting Institutions listed above contribute to the cost of publication of this Journal, but they are not owners or publishers and have no responsibility for its content or policies.

Mathematical papers intended for publication in the Pacific Journal of Mathematics should be in typed form or offset-reproduced (not dittoed), double spaced with large margins. Please do not use built up fractions in the text of the manuscript. However, you may use them in the displayed equations. Underline Greek letters in red, German in green, and script in blue. The first paragraph must be capable of being used separately as a synopsis of the entire paper. In particular it should contain no bibliographic references. Please propose a heading for the odd numbered pages of less than 35 characters. Manuscripts, in triplicate, may be sent to any one of the editors. Please classify according to the scheme of Math. Reviews, Index to Vol. 39. Supply name and address of author to whom proofs should be sent. All other communications should be addressed to the managing editor, or Elaine Barth, University of California, Los Angeles, California 90024.

There are page-charges associated with articles appearing in the Pacific Journal of Mathematics. These charges are expected to be paid by the author's University, Government Agency or Company. If the author or authors do not have access to such Institutional support these charges are waived. Single authors will receive 50 free reprints; joint authors will receive a total of 100 free reprints. Additional copies may be obtained at cost in multiples of 50 .

The Pacific Journal of Mathematics is issued monthly as of January 1966. Regular subscription rate: $\$ 190.00$ a year (5 Vols., 10 issues). Special rate: $\$ 95.00$ a year to individual members of supporting institutions.

Subscriptions, orders for numbers issued in the last three calendar years, and changes of address should be sent to Pacific Journal of Mathematics, P.O. Box 969, Carmel Valley, CA 93924, U.S.A. Old back numbers obtainable from Kraus Periodicals Co., Route 100, Millwood, NY 10546.

The Pacific Journal of Mathematics at P.O. Box 969, Carmel Valley, CA 93924 (ISSN 0030-8730) publishes 5 volumes per year. Application to mail at Second-class postage rates is pending at Carmel Valley, California, and additional mailing offices. Postmaster: send address changes to Pacific Journal of Mathematics, P.O. Box 969, Carmel Valley, CA 93924.

PUBLISHED BY PACIFIC JOURNAL OF MATHEMATICS, A NON-PROFIT CORPORATION

Copyright (C) 1986 by Pacific Journal of Mathematics 


\section{Pacific Journal of Mathematics}

\section{Vol. 123, No. $2 \quad$ April, 1986}

David Jay Anick, A loop space whose homology has torsion of all orders . . 257 Steven P. Diaz, Space curves that intersect often ................. 263

Thierry Fack and Hideki Kosaki, Generalized $s$-numbers of $\tau$-measurable

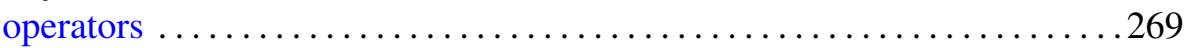

Karl Heinrich Hofmann and Karl Strambach, Lie's fundamental

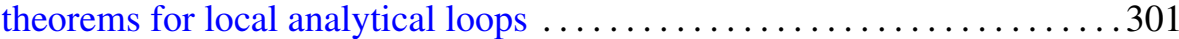

James Secord Howland, On the Kato-Rosenblum theorem ............. 329

Frieder Knüppel and Edzard Salow, Plane elliptic geometry over rings . . . . 337

Alan Noell, Peak points in boundaries not of finite type ................ 385

William J. Ralph, An extension of singular homology to Banach

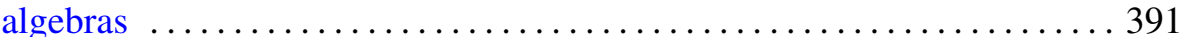

Wade C. Ramey, Averaging properties of pluriharmonic boundary values . . 407

Thomas Joseph Ransford, On the range of an analytic multivalued

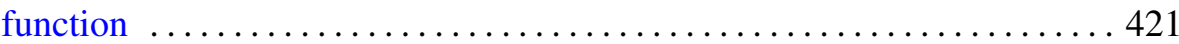

Christopher Donald Sogge, On restriction theorems of maximal-type . . . . 441

Edwin Spanier, Cohomology with supports $\ldots \ldots \ldots \ldots \ldots \ldots \ldots \ldots \ldots 47$

Emil J. Straube, Orthogonal projections onto subspaces of the harmonic

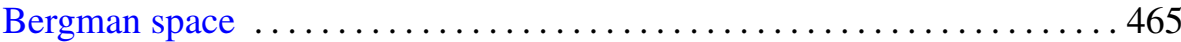

Thomas Vogel, Asymptotic behavior of two semilinear elliptic free boundary problems $\ldots \ldots \ldots \ldots \ldots \ldots \ldots \ldots \ldots$ 